\title{
EXTENDIBLE SETS IN PEANO ARITHMETIC
}

\author{
STUART T. SMITH
}

\begin{abstract}
Let $\mathscr{A}$ be a structure and let $U$ be a subset of $|\mathscr{A}|$. We say $U$ is extendible if whenever $\mathscr{B}$ is an elementary extension of $\mathscr{A}$, there is a $V \subseteq|\mathscr{B}|$ such that $(\mathscr{A}, U) \prec(\mathscr{B}, V)$. Our main results are: If $\mathscr{M}$ is a countable model of Peano arithmetic and $U$ is a subset of $|\mathscr{M}|$, then $U$ is extendible iff $U$ is parametrically definable in $\mathscr{M}$. Also, the cofinally extendible subsets of $|\mathscr{M}|$ are exactly the inductive subsets of $|\mathscr{M}|$. The end extendible subsets of $|\mathscr{M}|$ are not completely characterized, but we show that if $\mathscr{N}$ is a model of Peano arithmetic of arbitrary cardinality and $U$ is any bounded subset of $\mathcal{N}$, then $U$ is end extendible.
\end{abstract}

\section{INTRODUCTION}

In this paper, we are interested for the most part in models of Peano arithmetic. However, the main definition we require makes sense for any structure, with no restrictions on the language.

Definition 0.1. Let $\mathscr{A}$ be a structure and $U$ be a subset of $|\mathscr{A}|$.

(i) Suppose $\mathscr{B}$ is an elementary extension of $\mathscr{A}$. We say $U$ is extendible to $\mathscr{B}$ if there is a $V \subseteq|\mathscr{B}|$ such that $(\mathscr{A}, U) \prec(\mathscr{B}, V)$.

(ii) $U$ is extendible if $U$ is extendible to $\mathscr{B}$ whenever $\mathscr{B} \succ \mathscr{A}$.

(Here, of course, we have added to the language a new unary predicate symbol which is interpreted as $U$ in $\mathscr{A}$ and as $V$ in $\mathscr{B}$.) The term "elementary extendible" would be more precise, but it is also more cumbersome, and no ambiguity results from dropping the word "elementary".

Proposition 0.2. Let $U$ be a parametrically definable subset of $|\mathscr{A}|$. Then $U$ is extendible.

Our basic question is: What are the extendible subsets of a given structure (or more generally, of the models of a given theory)? For countable models of Peano arithmetic we have answered this question by showing that the converse to Proposition 0.2 holds.

Theorem 0.3. Let $\mathscr{M}$ be a countable model of Peano arithmetic and let $U$ be a subset of $|\mathscr{M}|$. Then $U$ is extendible $\Leftrightarrow U$ is parametrically definable in $\mathscr{M}$.

Received by the editors October 25, 1986 and, in revised form, May 10, 1988.

1980 Mathematics Subject Classification (1985 Revision). Primary 03C62, 03H 15. 
Proof. The nontrivial direction $(\Rightarrow)$ follows from two theorems which appear later in the paper. Suppose $U$ is a subset of $|\mathscr{M}|$ which is not parametrically definable in $\mathscr{M}$. If $U$ is inductive, Theorem 3.3 shows that $U$ is not extendible. If $U$ is not inductive, we can apply Theorem 4.15.

After some preliminaries in $\S 1$, we discuss extendibility in a general modeltheoretic context in $\S 2$. The remainder of the paper is devoted to models of Peano arithmetic. In view of the following Elementary Splitting Theorem (cf. [Smol]), we need consider only end and cofinal extensions, which we do in $\S \S 3$ and 4 , respectively.

Theorem 0.4. Suppose $\mathscr{M} \vDash \mathrm{PA}$ and $\mathscr{M} \prec \mathscr{N}$. There is a unique model $\mathscr{M}^{\prime}$ such that $\mathscr{M} \prec_{c} \mathscr{M}^{\prime} \prec_{e} \mathscr{N}$.

We show in $\S 3$ that if $\mathscr{M}$ is a model (of arbitrary cardinality) of PA and $U$ is a bounded subset of $\mathscr{M}$, then for any elementary end extension $\mathscr{N}$ of $\mathscr{M}$ we have $(\mathscr{M}, U) \prec(\mathscr{N}, U)$; we say that $U$ is end extendible. In $\S 4$ we complement a result obtained (independently of each other) by J. Schmerl [Sch] and H. Kotlarski [Ko] by showing that for countable $\mathscr{M}$, the cofinally extendible subsets of $|\mathscr{M}|$ are exactly the inductive subsets of $|\mathscr{M}|$.

In $\S 5$ we refine some of our results. We show that Theorem 0.3 still holds if we consider only simple elementary extensions. Similarly, Theorem 0.3 remains true if we require all the models to be recursively saturated.

The paper concludes with $\S 6$, in which we list some open questions.

\section{Preliminaries}

We deal first with arbitrary structures before specializing to models of Peano arithmetic; we let $\mathscr{A}, \mathscr{B}$ (with universes $A, B$ respectively) denote structures for any first-order language, and use $\mathscr{M}, \mathscr{N}$ (whose universes are $M, N$ respectively) to designate models of PA. The set of parametrically definable subsets of $A$ in $\mathscr{A}$ is denoted by $\operatorname{Def}(\mathscr{A})$. Where there is no ambiguity, we will be careless about distinguishing between predicate symbols and their interpretations in a structure.

We will continually make use of the following criterion for determining when an extension is elementary (cf. [CK]).

Proposition 1.1. Suppose $\mathscr{A} \subseteq \mathscr{B}$. Then $\mathscr{A} \prec \mathscr{B}$ if and only if for all formulas $\varphi\left(x, y_{1}, \ldots, y_{n}\right)$ and all $a_{1}, \ldots, a_{n} \in A$, if $\mathscr{B} \vDash \exists x \varphi\left(x, a_{1}, \ldots, a_{n}\right)$, then there is an $a \in A$ such that $\mathscr{B} \vDash \varphi\left(a, a_{1}, \ldots, a_{n}\right)$.

Sometimes we can show that an extension is elementary by demonstrating that the theory in question is model complete. Recall:

Definition 1.2. A theory $T$ is model complete if whenever $\mathscr{A}, \mathscr{B}$ are models of $T$ and $\mathscr{A} \subseteq \mathscr{B}$, then $\mathscr{A} \prec \mathscr{B}$.

The following theorem (which can be found in $[\mathrm{CK}]$ ) is useful. 
Theorem 1.3 (Lindström). Let $T$ be a theory in a countable language such that:

(i) All models of $T$ are infinite.

(ii) The union of any chain of models of $T$ is a model of $T$.

(iii) $T$ is $\kappa$-categorical for some infinite cardinal $\kappa$.

Then $T$ is model complete.

We now move on to models of Peano arithmetic. Suppose $\mathscr{M}$ and $\mathscr{N}$ are models of PA and $\mathscr{M} \subseteq \mathscr{N} . \mathscr{N}$ is an end extension of $\mathscr{M}\left(\mathscr{M} \subseteq_{e} \mathscr{N}\right)$ if

$$
(\forall a \in M)(\forall b \in N \backslash M)(a<b) .
$$

$\mathscr{N}$ is a cofinal extension of $\mathscr{M}\left(\mathscr{M} \subseteq_{c} \mathscr{N}\right)$ if

$$
(\forall b \in N)(\exists a \in M)(b<a) .
$$

We write $\mathscr{M} \prec_{e} \mathscr{N}$ and $\mathscr{M} \prec_{c} \mathscr{N}$ if the extensions are also elementary. $\mathscr{N}$ is a simple extension of $\mathscr{M}$ if there exists a $b \in N$ such that every element of $N$ is definable in $\mathscr{N}$ with parameters from $M \cup\{b\}$. We write $\mathscr{N}=\mathscr{M}(b)$.

It is convenient to use the usual notation for intervals in $\mathscr{M}$; thus $[a, b)=$ $\{m \in M: a \leq m<b\}$, etc. A subset $C \subseteq M$ is a class in $\mathscr{M}$ if for every $a \in M, C \cap[0, a) \in \operatorname{Def}(\mathscr{M}) . C$ is a proper class if $C$ is a class but $C \notin$ $\operatorname{Def}(\mathscr{M})$.

We code $\mathscr{M}$-finite sequences in $\mathscr{M}$ in one of the usual ways; e.g. we could take $(x)_{y}$ to be the exponent of the $y$ th prime $p_{y}$ in the prime factorization of $x$.

A subset $U \subseteq M$ is inductive if the expansion $(\mathscr{M}, U)$ satisfies full induction in the expanded language. One can prove (by induction on $a \in M$ in the definition of class, and using the codability of $\mathscr{M}$-finite sets) that every inductive set is a class.

We now borrow some terminology from [KS]. A subset $I \subseteq M$ is a cut of $\mathscr{M}$ if $I$ is a nonempty initial segment with no greatest element. The standard cut is simply $\omega$. Suppose $I$ is a cut of $\mathscr{M}$ and $\mathscr{M} \prec \mathscr{N}$. Then $\mathscr{N}$ fills $I$ with $b$ if $b \in N$ and $i<b<j$ for all $i \in I$ and $j \in M \backslash I$.

Let $\tau\left(x, y_{1}, \ldots, y_{n}\right)$ be a set of (parameter-free) formulas with at most $x, y_{1}, \ldots, y_{n}$ free such that the set of Gödel numbers of the formulas in $\tau$ is recursive. If $a_{1}, \ldots, a_{n} \in M$ are such that $\tau\left(x, a_{1}, \ldots, a_{n}\right)$ is finitely satisfiable, we say that $\tau(x, \vec{a})$ is a recursive type over $\mathscr{M} . \mathscr{M}$ is recursively saturated if every recursive type $\tau(x, \vec{a})$ over $\mathscr{M}$ is realized by some $b \in M$; that is, $\mathscr{M} \vDash \tau(b, \vec{a})$ for some $b \in M$.

We have some latitude in our choice of language $L$ for PA. Theorem 0.3 , and Theorems 3.3 and 4.15 on which it depends, are true for any countable language $L$ extending the usual language of PA, provided we assume full $L$-induction. For Theorems 3.11 and 3.14 we must be more careful, since our proofs are ultimately based on the existence of a satisfaction definition for $\Sigma_{n}$-formulas (necessary for Lemma 3.5). When we discuss recursively saturated models in $\S 5$, the language must be effectively presented. 


\section{EXTENDIBILITY IN A GENERAL SETTING}

In this section we discuss arbitrary $L$-structures, where $L$ is any first-order language. For convenience, we record some simple properties of extendibility in the following proposition.

Proposition 2.1. Suppose $\mathscr{A}$ and $\mathscr{B}$ are L-structures, $\mathscr{A} \prec \mathscr{B}$, and the subset $U \subseteq A$ is extendible to $\mathscr{B}$.

(i) If $L^{\prime}$ is a sublanguage of $L$ and $\mathscr{A}^{\prime}, \mathscr{B}^{\prime}$ are the corresponding reducts to $L^{\prime}$, then $U \subseteq\left|\mathscr{A}^{\prime}\right|$ is extendible to $\mathscr{B}^{\prime}$.

(ii) Let $W \subseteq \mathscr{A}$ be any set parametrically definable in the expansion $(\mathscr{A}, U)$. Then $W$ is extendible to $\mathscr{B}$.

(iii) Let $\left\{P_{\alpha}: \alpha<\kappa\right\}$ be an arbitrary collection of new predicate symbols such that each $P_{\alpha}^{\mathscr{Q}} \in \operatorname{Def}(\mathscr{A})$. Then $U$, considered as a subset of the universe of $\left(\mathscr{A},\left\{P_{\alpha}^{\mathscr{A}}\right\}_{\alpha<\kappa}\right\}$, is extendible to the expansion $\left(\mathscr{B},\left\{P_{\alpha}^{\mathscr{B}}\right\}_{\alpha<\kappa}\right)$.

The definition of extendibility lends itself to alteration in various ways, as in the following:

Definition 2.2. Let $\mathscr{A}$ be a structure and let $U$ be a subset of $A$.

(i) $U$ is weakly extendible if there exists a proper elementary extension $\mathscr{B}$ of $\mathscr{A}$ such that $U$ is extendible to $\mathscr{B}$.

(ii) Suppose $\mathscr{B}$ is an elementary extension of $\mathscr{A}$. We say $U$ is uniquely extendible to $\mathscr{B}$ if there is a unique $V \subseteq B$ such that $(\mathscr{A}, U) \prec(\mathscr{B}, V) . U$ is uniquely extendible if $U$ is uniquely extendible to $\mathscr{B}$ whenever $\mathscr{B} \succ \mathscr{A}$.

Clearly unique extendibility implies extendibility, and for infinite structures we also have that extendibility implies weak extendibility. Note also that parts (ii) and (iii) of Proposition 2.1 hold if we replace the word "extendible" by "uniquely extendible".

The next proposition shows that Definition 2.2 enables us to characterize the weakly extendible and the uniquely extendible subsets of an infinite structure $\mathscr{A}$.

Proposition 2.3. Let $\mathscr{A}$ be an infinite structure.

(i) Every subset $U$ of $A$ is weakly extendible.

(ii) $A$ subset $U$ of $A$ is uniquely extendible $\Leftrightarrow U$ is parametrically definable in $\mathscr{A}$.

Proof. (i) By compactness (or by Löwenheim-Skolem) $(\mathscr{A}, U$ ) has a proper elementary extension $(\mathscr{B}, V)$.

(ii) $(\Leftarrow)$ Trivial.

$(\Rightarrow)$ This follows immediately from Beth's Definability Theorem (cf. [CK]), where we take $\Sigma(U)$ to be the set

$$
\Sigma(U)=\operatorname{Th}(\mathscr{A}, U, a)_{a \in A} .
$$

Definition 2.2 will prove to be more fruitful when we restrict the allowable class of elementary extensions, as in $\S \S 3$ and 4. 
For a given structure $\mathscr{A}$, let $\mathscr{E}(\mathscr{A})$ denote the collection of extendible subsets of $\mathscr{A}$. It follows that

$$
\operatorname{Def}(\mathscr{A}) \subseteq \mathscr{E}(\mathscr{A}) \subseteq \mathscr{P}(\mathscr{A}),
$$

where $\mathscr{P}(\mathscr{A})$ denotes the power set of the universe of $\mathscr{A}$. Supposing that $\mathscr{A}$ is infinite and that the language is countable (so $\operatorname{Def}(\mathscr{A}) \neq \mathscr{P}(\mathscr{A})$ ), we note that exactly one of the following conditions holds for $\mathscr{A}$ :

(i) $\mathscr{E}(\mathscr{A})=\mathscr{P}(\mathscr{A})$,

(ii) $\operatorname{Def}(\mathscr{A}) \subsetneq \mathscr{E}(\mathscr{A}) \subsetneq \mathscr{P}(\mathscr{A})$,

(iii) $\mathscr{E}(\mathscr{A})=\operatorname{Def}(\mathscr{A})$.

Our next task is to provide examples of structures satisfying each of these conditions in turn.

Proposition 2.4. Let $L$ be a language consisting of finitely many unary predicate symbols $P_{1}, \ldots, P_{k}$, and let $\mathscr{A}$ be an infinite L-structure. Then $\mathscr{E}(\mathscr{A})=$ $\mathscr{P}(\mathscr{A})$, i.e. every subset of $A$ is extendible.

Proof. First let us introduce $2^{k}$ new unary predicate symbols $\left\{R_{\sigma}: \sigma \in S\right\}$, where each $\sigma$ is a sequence of the form $\left\langle\varepsilon_{1}, \ldots, \varepsilon_{k}\right\rangle$ in which each $\varepsilon_{i}$ is either +1 or -1 . We define the $R_{\sigma}$ 's in terms of the $P_{i}$ 's as follows:

$$
R_{\sigma}(x)=R_{\left\langle\varepsilon_{1}, \ldots, \varepsilon_{k}\right\rangle}(x) \equiv \varepsilon_{1} P_{1}(x) \wedge \varepsilon_{2} P_{2}(x) \wedge \cdots \wedge \varepsilon_{k} P_{k}(x),
$$

where $(+1) P$ is taken to be $P$ and $(-1) P$ is $\neg P$.

Then the sets $R_{\sigma}^{\mathscr{S}}$ partition $A$, although one or more of the partitions might be empty. Since $\mathscr{A}$ is infinite, at least one $R_{\sigma}^{\mathscr{A}}$ is also infinite.

Each $P_{i}$ is definable from the $R_{\sigma}$ 's. By Proposition 2.1, it is enough to show that every subset of $A$ is extendible in the $L^{\prime}$-structure $\mathscr{A}^{\prime}=\left(A, R_{\sigma}^{\mathscr{A}}\right)_{\sigma \in S}$, where $L^{\prime}=\left\{R_{\sigma}: \sigma \in S\right\}$.

Let $U \subseteq A$ be arbitrary. We let $T$ be the theory in the language $L^{\prime} \cup\{U\}$ axiomatized as follows:

(i) Given $\sigma \in S$, if $U \cap R_{\sigma}^{\mathscr{U}}$ is finite of cardinality $n \geq 0$, then $T$ contains the sentence "There are exactly $n x$ 's such that $U(x) \wedge R_{\sigma}(x)$ ".

(ii) If $U \cap R_{\sigma}^{\sigma /}$ is infinite, then $T$ contains all sentences of the form "There are at least $n \quad x$ 's such that $U(x) \wedge R_{\sigma}(x)$ " for all $n \in \omega$.

(iii) If $(A \backslash U) \cap R_{\sigma}^{i v}$ is finite of cardinality $n \geq 0, T$ contains the sentence "There are exactly $n \quad x$ 's such that $\neg U(x) \wedge R_{\sigma}(x)$ ".

(iv) If $(A \backslash U) \cap R_{\sigma}^{\sigma /}$ is infinite, $T$ contains all sentences "There are at least $n \quad x$ 's such that $\neg U(x) \wedge R_{\sigma}(x)$ " for all $n \in \omega$.

Since at least one of the partitions $R_{\sigma}^{\sigma}$ is infinite, we see that all models of $T$ are infinite. The union of any chain of models of $T$ is clearly a model of $T$, and $T$ is $\aleph_{0}$-categorical; by Theorem 1.3,T is model complete.

Now suppose $\mathscr{B}^{\prime}$ is an $L^{\prime}$-structure such that $\mathscr{A} \prec \mathscr{B}^{\prime}$. It suffices (by the model completeness of $T$ ) to find a $V \subseteq B$ such that $\left(\mathscr{A}^{\prime}, U\right) \subseteq\left(\mathscr{B}^{\prime}, V\right)$ and 
$\left(\mathscr{B}^{\prime}, V\right) \vDash T$. For each $\sigma \in S$, let $U_{\sigma}=U \cap R_{\sigma}^{\alpha^{\prime}}$ and define $V_{\sigma} \subseteq R_{\sigma}^{\not \not^{\prime}}$ as follows:

(i) If $U_{\sigma}$ is finite, take $V_{\sigma}=U_{\sigma}$.

(ii) If $R_{\sigma}^{\left(W^{\prime}\right.} \backslash U_{\sigma}$ is finite, take $V_{\sigma} \subseteq R_{\sigma}^{\beta^{\prime}}$ such that $R_{\sigma}^{\mathscr{B}^{\prime}} \backslash V_{\sigma}=R_{\sigma}^{\mathscr{S} \mathcal{L}^{\prime}} \backslash U_{\sigma}$.

(iii) If $U_{\sigma}$ and $R_{\sigma}^{\mathscr{V}^{\prime}} \backslash U_{\sigma}$ are both infinite, we can choose $V_{\sigma} \subseteq R_{\sigma}^{\mathscr{B}^{\prime}}$ arbitrarily so long as $U_{\sigma} \subseteq V_{\sigma}$ and $R_{\sigma}^{\sigma^{\prime}} \backslash U_{\sigma} \subseteq R_{\sigma}^{\not^{\prime}} \backslash V_{\sigma}$.

We now take $V=\bigcup_{\sigma \in S} V_{\sigma}$. It is easy to check that $\left(\mathscr{A}^{\prime}, U\right) \subseteq\left(\mathscr{B}^{\prime}, V\right)$ and $\left(\mathscr{B}^{\prime}, V\right) \vDash T$. Thus the arbitrary set $U$ is extendible to $\mathscr{B}^{\prime}$ and therefore to $\mathscr{B}$, hence $\mathscr{E}(\mathscr{A})=\mathscr{P}(\mathscr{A})$.

We are grateful to Matatyahu Rubin for suggesting consideration of the following example.

Proposition 2.5. Let $\mathscr{A}$ be a dense linear ordering without endpoints, where we take the language to be $L=\{<\}$. Then $\operatorname{Def}(\mathscr{A}) \subsetneq \mathscr{E}(\mathscr{A}) \subsetneq \mathscr{P}(\mathscr{A})$.

Proof. We first remark that the theory of dense linear orderings without endpoints is model complete, so any extension of $\mathscr{A}$ to another d.l.o. without endpoints is automatically elementary.

Let us begin by showing that $\mathscr{E}(\mathscr{A}) \subsetneq \mathscr{P}(\mathscr{A})$. This is a consequence of the following lemma.

Lemma 2.6. Let $u_{0}<u_{1}<\cdots<u_{n}<\cdots \quad(n \in \omega)$ be an increasing $\omega$-sequence of elements of $A$. Then the set $U=\left\{u_{n}: n \in \omega\right\}$ is not extendible.

Proof. Let $X=\left\{a \in A: \exists u_{n} \in U\left(a<u_{n}\right)\right\}$, and let $(I,<)$ be a dense linear ordering with least element $c$ and no greatest element; we assume that $A \cap I=\varnothing$. We define a new dense linear ordering $\mathscr{B}$ as follows: $B=A \cup I$, and the given orderings on $A$ and $I$ are extended so that $X<I<(A \backslash X)$; i.e., $a_{1}<i<a_{2}$ whenever $a_{1} \in X, i \in I$, and $a_{2} \in A \backslash X$. (Note that $A \backslash X$ might be the empty set.)

Clearly $\mathscr{B}$ is a dense linear ordering without endpoints and $\mathscr{A} \subseteq \mathscr{B}$; by our earlier remark, $\mathscr{A} \prec \mathscr{B}$. We show that there is no $V \subseteq B$ such that $(\mathscr{A}, U) \prec(\mathscr{B}, V)$. Any such $V$ would have to be of the form $U \cup D$ for some subset $D \subseteq I$; we assume that such a $D$ exists and derive a contradiction.

Case 1. Suppose $D=\varnothing$. Then $V=U$ has supremum $c$ (the least element of $I)$ in $\mathscr{B}$, whereas $U$ has either a different supremum or no supremum at all in $\mathscr{A}$. This contradicts the assumption that $(\mathscr{A}, U) \prec(\mathscr{B}, V)$.

Case 2. If $D \neq \varnothing$ and $(\mathscr{A}, U) \prec(\mathscr{B}, V)$, then the least element $c$ of $I$ has the following property: there are elements of $V$ less than $c$ and there is at least one element of $V$ greater than or equal to $c$, but the set of elements of $V$ less than $c$ has no greatest member. However, there is no $a \in A$ exhibiting this property with respect to $U$ in $(\mathscr{A}, U)$-this contradicts the assumption that $(\mathscr{A}, U) \prec(\mathscr{B}, V)$. 
Next we demonstrate that $\operatorname{Def}(\mathscr{A}) \subsetneq \mathscr{E}(\mathscr{A})$. One can show (e.g. by elimination of quantifiers) that the parametrically definable subsets of $\mathscr{A}$ are those sets $X \subseteq A$ such that $X$ is the union of finitely many intervals (closed, halfopen, or open) in $\mathscr{A}$ and finitely many singleton sets $\{a\}$ with $a \in A$. Thus the following lemma and corollary complete the proof of Proposition 2.5.

Lemma 2.7. Let $U \subseteq A$ be such that $U$ is both dense and codense in $\mathscr{A}$. (We say that $U$ is codense in $\mathscr{A}$ if $A \backslash U$ is dense in $\mathscr{A}$.) Then $U$ is an extendible subset of $A$.

Proof. Let $T$ be the theory in the language $L \cup\{U\}$ obtained by adding the following axiom to the axioms for a dense linear ordering without endpoints:

$$
\forall x \forall y[x<y \rightarrow \exists z \exists w(x<z<y \wedge x<w<y \wedge U(z) \wedge \neg U(w))] .
$$

This axiom states that $U$ is dense and codense in $\mathscr{A}$. A routine back-and-forth argument shows that $T$ is $\aleph_{0}$-categorical. Clearly $T$ also satisfies conditions (i) and (ii) of Theorem 1.3, so $T$ is model complete.

Let $\mathscr{B}$ be any elementary extension of $\mathscr{A}$; by the above, it suffices to find a subset $V \subseteq B$ such that $(\mathscr{A}, U) \subseteq(\mathscr{B}, V)$ and $(\mathscr{B}, V) \vDash T$. We show that this is always possible by proving the following statement:

(*) Let $\mathscr{B}=(B,<)$ be a dense linear ordering without endpoints, and let $A \subseteq B$ be either the empty set or such that $\mathscr{A}=(A,<)$ is a dense linear ordering without endpoints. Let $U \subseteq A$ be empty if $A=\varnothing$, and such that $U$ is dense and codense in $\mathscr{A}$ otherwise. Then there is a $V \subseteq B$ dense and codense in $\mathscr{B}$ such that $V \cap A=U$.

We prove $(*)$ by induction on the (infinite) cardinality of $B$. If $\operatorname{card}(B)=\aleph_{0}$ the argument is straightforward and corresponds to Stage 2 in the inductive step which follows.

Now take $\kappa>\aleph_{0}$ and assume $(*)$ holds for all $\mathscr{B}$ such that $\aleph_{0} \leq \operatorname{card}(B)<$ $\kappa$. Let $\mathscr{B}$ be a dense linear ordering without endpoints such that $\operatorname{card}(B)=\kappa$. Let $C=\left\{I_{\alpha}: \alpha<\lambda\right\}$ enumerate all open intervals in $\mathscr{B}$ such that $\operatorname{card}\left(I_{\alpha}\right)<$ $\kappa$, and let $D=\left\{J_{\beta}: \beta<\mu\right\}$ enumerate all open intervals in $\mathscr{B}$ such that $\operatorname{card}\left(J_{\beta}\right)=\kappa$. Here $\lambda, \mu$ are cardinals such that $0 \leq \lambda, \mu \leq \kappa$ and at least one of them is equal to $\kappa$.

Let $A$ and $U$ satisfy the hypotheses in (*). We show how to extend $U$ to a suitable $V \subseteq B$.

Stage 1. We extend $U$ to $A \cup(\cup C)$. Let $\alpha<\lambda$ and assume inductively that for each $\gamma<\alpha$ we have defined a subset $W_{\gamma} \subseteq I_{\gamma}$ which is dense and codense in $I_{\gamma}$ and such that

$$
W_{\gamma} \cap I_{\delta}=W_{\delta} \cap I_{\gamma} \quad \text { whenever } \delta<\gamma
$$

and $W_{y} \cap A=I_{y} \cap U$.

Now $A \cup\left(\bigcup_{y<\alpha} I_{y}\right)$ is a dense linear ordering without endpoints (unless $\alpha=0$ and $A=\varnothing$ in which case it is the empty set). Clearly $U \cup\left(\bigcup_{\gamma<r} W_{\gamma}\right)$ is dense 
in $A \cup\left(\bigcup_{y<r} I_{y}\right)$; to see that it is also codense, note that it suffices to show that whenever $\delta<\gamma<\alpha$, we have not included any elements of $I_{\delta} \backslash W_{\delta}$ or of $A \backslash U$-in $W_{\gamma}$. But by the induction hypothesis, $W_{\gamma} \cap I_{\delta} \subseteq W_{\delta}$, hence $W_{\gamma} \cap \cap\left(I_{\delta} \backslash W_{\delta}\right)=\varnothing$. Similarly, $W_{\gamma} \cap A \subseteq U$ so $W_{y} \cap(A \backslash U)=\varnothing$.

Consider $I_{\alpha} \cap\left[A \cup\left(\bigcup_{i<\alpha} I_{\gamma}\right)\right]$. This set is either empty, or consists of a single point, or else it is a dense linear ordering (with 0,1 , or 2 endpoints). In the last case, $I_{\alpha} \cap\left[A \cup\left(\bigcup_{\gamma<\alpha} I_{\gamma}\right)\right]$ has $I_{\alpha} \cap\left[U \cup\left(\bigcup_{\gamma<\alpha} W_{\gamma}\right)\right]$ as a dense, codense subset. Since $\operatorname{card}\left(I_{\alpha}\right)<\kappa$ and isolated points pose no problems, we can apply the induction hypothesis $(*)$ to extend $I_{\alpha} \cap\left[U \cup\left(\bigcup_{\gamma<\alpha} W_{\gamma}\right)\right]$ to a subset $W_{\alpha} \subseteq I_{\alpha}$ which is dense and codense in $I_{\alpha}$, and such that

$$
W_{\alpha} \cap\left[A \cup\left(\bigcup_{\gamma<\alpha} I_{\alpha}\right)\right]=I_{\alpha} \cap\left[U \cup\left(\bigcup_{\gamma<\alpha} W_{\alpha}\right)\right] .
$$

If we take the intersection of both sides of this equation with $I_{\delta}$, where $\delta<\alpha$, we obtain

$$
\begin{aligned}
W_{\alpha} \cap I_{\delta} & =I_{\alpha} \cap\left[\left(U \cap I_{\delta}\right) \cup\left(\bigcup_{\gamma<\alpha}\left(W_{\gamma} \cap I_{\delta}\right)\right)\right] \\
& =I_{\alpha} \cap\left[\left(U \cap I_{\delta}\right) \cup\left(\bigcup_{\gamma<\alpha}\left(W_{\delta} \cap I_{\gamma}\right)\right)\right] \\
& =I_{\alpha} \cap\left[\left(U \cap I_{\delta}\right) \cup W_{\delta}\right]=I_{\alpha} \cap W_{\delta}
\end{aligned}
$$

as required, where we have used the fact that $W_{\delta} \cap I_{\gamma}=W_{\delta}$ when $\gamma=\delta$, and is a subset of $W_{\delta}$ otherwise, to get that $\bigcup_{\gamma<x}\left(W_{\delta} \cap I_{\gamma}\right)=W_{\delta}$. We also use the induction hypothesis to replace $W_{\gamma} \cap I_{\delta}$ by $W_{\delta} \cap I_{\gamma}$, and to show that $U \cap I_{\delta} \subseteq W_{\delta}$. A similar proof verifies that $W_{\alpha} \cap A=I_{\alpha} \cap U$. This completes the induction.

Let $W=U \cup\left(\bigcup_{a<i} W_{\alpha}\right)$. Then $W$ is a dense, codense subset of $A \cup(\cup C)$ and $W \cap A=U$.

If $B=A \cup(\cup C)$ we can stop here, but in general we must go on to:

Stage 2. We extend $W$ to $A \cup(\cup C) \cup(\cup D)=B$. Let $\beta<\mu$ and assume inductively that for each $\delta<\beta$ we have defined $x_{\delta}, y_{\delta} \in J_{\delta}$ such that $x_{\delta} \notin$ $(A \cup(\cup C)) \backslash W$ and $y_{\delta} \notin W$ for all $\delta<\beta$, and $\left\{x_{\delta}: \delta<\beta\right\} \cap\left\{y_{\delta}: \delta<\beta\right\}=\varnothing$.

Consider the interval $J_{\beta}$ and in particular the intersection $J_{\beta} \cap(A \cup(\cup C))$. If there are two elements in this intersection, then there are infinitely many, hence there are also infinitely many elements in the sets $J_{\beta} \cap W$ and $J_{\beta} \cap$ $(A \cup(\cup C)) \backslash W$. Choose $x_{\delta}$ arbitrarily from the former set and $y_{\delta}$ from the latter in this case.

Now suppose $\operatorname{card}\left(J_{\beta} \cap(A \cup(\cup C))\right) \leq 1$. Since $\operatorname{card}\left(J_{\beta}\right)=\kappa$ and $\beta<\mu \leq$ $\kappa$, there are $\kappa$ many elements in the set

$$
J_{\beta} \backslash\left[A \cup(\bigcup C) \cup\left\{x_{\delta}: \delta<\beta\right\} \cup\left\{y_{\delta}: \delta<\beta\right\}\right] .
$$


Let $x_{\beta} \neq y_{\beta}$ be two arbitrary elements of this set. Then $x_{\beta}$ and $y_{\beta}$ have the requisite properties and the induction is complete.

Finally, let $V \subseteq B$ be any set satisfying the properties $W \cup\left\{x_{\beta}: \beta<\mu\right\} \subseteq V$ and $\left\{y_{\beta}: \beta<\mu\right\} \cup[(A \cup(\cup C)) \backslash W] \subseteq B \backslash V$. Then every open interval $I_{\alpha}$ or $J_{\beta}$ contains an element of $V$ and an element of $B \backslash V$, and $V \cap A=U$.

Corollary 2.8. Let $\mathscr{A}=\langle A,<\rangle$ be a dense linear ordering without endpoints. Then $\mathscr{A}$ has a dense, codense subset $U$.

Proposition 2.1(ii) implies that $\mathscr{E}(\mathscr{A})$ is closed under complementation for any structure $\mathscr{A}$. However, we can now show that $\mathscr{E}(\mathscr{A})$ need not be a field of sets.

Corollary 2.9. Let $\mathscr{A}=\langle A,<\rangle$ be a dense linear ordering without endpoints. Then $\mathscr{E}(\mathscr{A})$ is not closed under unions or intersections.

Proof. Let $U$ be a dense, codense subset of $\mathscr{A}$ and let $s_{0}<s_{1}<\cdots<s_{n}<\cdots$ $(n \in \omega)$ be an increasing $\omega$-sequence of elements of $U$; take $S=\left\{s_{n}: n \in \omega\right\}$ and $W=(A \backslash U) \cup S$. Then $W$ is also dense and codense in $\mathscr{A}$. Thus $U$ and $W$ are extendible by Lemma 2.7, whereas $U \cap W=S$ is not by Lemma 2.6. Taking complements shows that $\mathscr{E}(\mathscr{A})$ is not closed under union either.

We complete our series of examples with the following:

Proposition 2.10. Let $\mathbb{N}$ denote the standard model of arithmetic, formulated in any countable first-order $L$ which includes the usual language of arithmetic. Then $\mathscr{E}(N)=\operatorname{Def}(\mathbb{N})$.

Proof. In fact more is true: If $\mathscr{M}$ is any proper conservative elementary extension of $\mathbb{N}$, then the only subsets of $\mathbb{N}$ which are extendible to $\mathscr{M}$ are the parametrically definable ones. This follows from Theorem 3.3 in the next section, if we note that every subset of $\mathbb{N}$ is an inductive class.

\section{END EXTENDIBILITY}

We restrict our attention to end extensions of models of Peano arithmetic in this section; accordingly, we modify Definition 0.1 as follows:

Definition 3.1. Let $\mathscr{M}$ be a model of PA and $U \subseteq M . U$ is end extendible if $U$ is extendible to $\mathscr{N}$ whenever $\mathscr{M} \prec_{e} \mathscr{N}$.

Our goal in this section is to describe the set of end extendible subsets of $\mathscr{M}$. We fall short of a complete characterization, but Theorems 3.3 and 3.14 give us substantial information. We make no assumptions regarding the cardinality of $\mathscr{M}$.

An extension $\mathscr{M} \subseteq \mathscr{N}$ is conservative if for all $X \in \operatorname{Def}(\mathscr{N}), X \cap M \in$ $\operatorname{Def}(\mathscr{M})$. Conservative extensions are automatically end extensions. The basic 
existence result for conservative extensions is:

Theorem 3.2 (MacDowell-Specker [MS]). Every $\mathscr{M} \vDash$ PA has a proper conservative elementary end extension.

The MacDowell-Specker Theorem has the following immediate consequence.

Theorem 3.3. Let $\mathscr{M} \vDash \mathrm{PA}$ and let $\mathscr{N}$ be a proper conservative elementary end extension of $\mathscr{M}$. Let $U \subseteq M$ be a proper class in $\mathscr{M}$. Then $U$ is not extendible to $\mathscr{N}$. In particular, no undefinable inductive subset of $M$ is extendible to $\mathscr{N}$. Proof. The fact that $U$ is a class means that

$$
(\mathscr{M}, U) \vDash \forall x \exists y \forall z<x\left(U(z) \leftrightarrow p_{z} \mid y\right),
$$

where $p_{z}$ is the $z$ th prime number and $p_{z} \mid y$ means $p_{z}$ divides $y$. If $U$ were extendible to some $V \subseteq N$, we would also have

$$
(\mathscr{N}, V) \vDash \forall x \exists y \forall z<x\left(V(z) \leftrightarrow p_{z} \mid y\right),
$$

so $V$ would also have to be a class. Take $b \in N \backslash M$. Then $V \cap[0, b) \in \operatorname{Def}(\mathcal{N})$. Since the extension is conservative, we must have

$$
V \cap[0, b) \cap M \in \operatorname{Def}(\mathscr{M}) .
$$

But this set is $V \cap M=U$, which is a proper class by assumption. This contradiction shows that no such $V$ exists.

In particular, the end extendible subsets of $\mathbb{N}$ are precisely the parametrically definable sets. We will show that $\mathbb{N}$ is the only model of PA with this property.

Let $L$ be the language for Peano arithmetic which, besides containing the usual symbols $+, \cdot, 0,1,<$, also contains a two-place function symbol $\beta$ for some definable pairing function, and one-place function symbols $\pi_{1}$ and $\pi_{2}$ such that $\pi_{1}(\beta(x, y))=x$ and $\pi_{2}(\beta(x, y))=y$. As before, we will work with certain expansions of $L$ as well as with $L$ itself. We must therefore be somewhat careful in our terminology; for example, we need to distinguish between $\Sigma_{n}$ - and $\exists_{n}$-formulas.

Definition 3.4. Let $L^{\prime}$ be an expansion of $L$ and let $\mathscr{M} \vDash$ PA be an $L^{\prime}$-structure.

(i) $\exists_{0} \quad\left(=\forall_{0}\right)$ denotes the set of $L^{\prime}$-formulas with parameters from $M$ (henceforth denoted by $L^{\prime}(\mathscr{M})$ ) which are logically equivalent to open formulas.

(ii) $\exists_{n+1}\left[\forall_{n+1}\right]$ denotes the set of $L^{\prime}(\mathscr{M})$-formulas $\varphi$ such that $\varphi$ is logically equivalent to a formula of the form $\exists x \psi\left(x, y_{1}, \ldots, y_{k}\right)$ [resp. $\left.\forall x \psi\left(x, y_{1}, \ldots, y_{k}\right)\right]$, where $\psi\left(x, y_{1}, \ldots, y_{k}\right)$ is in $\forall_{n}$ [resp. in $\exists_{n}$ ].

(iii) For all $n \in \omega, D_{n}=\exists_{n} \cap \forall_{n}$.

(iv) $\Sigma_{n}, \Pi_{n}$, and $\Delta_{n}$ are defined in a corresponding way to $\exists_{n}, \forall_{n}$, and $D_{n}$, respectively, the only difference being that $\Sigma_{0}\left(=\Pi_{0}=\Delta_{0}\right)$ is the set of all $L^{\prime}(\mathscr{M})$-formulas logically equivalent to $L^{\prime}(\mathscr{M})$-formulas having only bounded quantifiers. 
In $L$, this distinction is unnecessary since the Matijasevic theorem tells us that $\Sigma_{n}=\exists_{n}$ for all $n>0$. For this reason we will use the $\Sigma_{n}$-hierarchy when dealing with $L$, and the $\exists_{n}$-hierarchy in connection with expansions of $L$.

It is well known (cf. [Smo2], for example) that for each $n \in \omega$ there is a definable satisfaction relation for $\Sigma_{n}$-formulas in PA. More precisely, given $n \in$ $\omega$, there is for each $k \in \omega$ a formula $\operatorname{Sat}_{\Sigma_{n}}^{k}\left(x, y_{1}, y_{2}, \ldots, y_{k}\right)$ such that for any $\Sigma_{n}$-formula $\varphi\left(y_{1}, \ldots, y_{k}\right)$ of $L$ whose free variables are among $y_{1}, \ldots, y_{k}$, we have

$$
\mathrm{PA} \vdash \forall y_{1} \cdots \forall y_{k}\left[\varphi\left(y_{1}, \ldots, y_{k}\right) \leftrightarrow \operatorname{Sat}_{\Sigma_{n}}^{k}\left(\left\ulcorner\varphi^{\urcorner}, y_{1}, \ldots, y_{k}\right)\right] .\right.
$$

(Here $\left.{ }^{\ulcorner} \varphi\right\urcorner$ is the Gödel number of $\varphi$ according to some recursive assignment of Gödel numbers.) Using Overspill we can conclude the following:

Lemma 3.5 [Smo2]. Let $\mathscr{M} \vDash \mathrm{PA}$ be nonstandard and let $n \in \omega$. Then every recursive $\Sigma_{n}$-type over $\mathscr{M}$ is realized in $\mathscr{M}$.

We can of course replace $\Sigma_{n}$ by $\Pi_{n}$ or $\Delta_{n}$ here. So if we confine our attention to recursive types of bounded complexity, every nonstandard $\mathscr{M}$ behaves like a recursively saturated model. We thus expect some properties of recursively saturated models to be mirrored in arbitrary nonstandard models of PA.

For instance, a basic fact about all recursively saturated models is that they are $\omega$-homogeneous (see [BS]). The corresponding result about arbitrary nonstandard models appears in Lemma 3.7.

Definition 3.6. Let $\mathscr{M} \vDash \mathrm{PA}$ and let $n \in \omega$.

(i) Let $a_{1}, \ldots, a_{k}, b_{1}, \ldots, b_{l} \in M$. The $\Sigma_{n}$-type of $a_{1}, \ldots, a_{k}$ over $b_{1}$, $\ldots, b_{l}$ is the set of all $\Sigma_{n}$-formulas $\varphi\left(x_{1}, \ldots, x_{k}, b_{1}, \ldots, b_{l}\right)$ in $L \cup\left\{b_{1}, \ldots\right.$, $b_{l}$ \} such that

$$
\mathscr{M} \vDash \varphi\left(a_{1}, \ldots, a_{k}, b_{1}, \ldots, b_{l}\right) .
$$

(ii) Let $a_{1}, \ldots, a_{k}, c_{1}, \ldots, c_{k} \in M$. We write $\left(\mathscr{M}, a_{1}, \ldots, a_{k}\right) \equiv_{\Sigma_{n}}(\mathscr{M}$, $c_{1}, \ldots, c_{k}$ ) if $a_{1}, \ldots, a_{k}$ and $c_{1}, \ldots, c_{k}$ have the same $\Sigma_{n}$-type (over $\varnothing$ ). (Similarly for $\Pi_{n}$ and $\Delta_{n}$.)

Note that $a_{1}, \ldots, a_{k}$ and $c_{1}, \ldots, c_{k}$ have the same $\Sigma_{n}$-type over $b_{1}, \ldots, b_{l}$ iff $b_{1}, \ldots, b_{l}, a_{1}, \ldots, a_{k}$ and $b_{1}, \ldots, b_{l}, c_{1}, \ldots, c_{k}$ have the same $\Sigma_{n}$-type (over $\varnothing$ ).

Lemma 3.7. Let $\mathscr{M} \vDash \mathrm{PA}, n \geq 1$, and suppose $a_{1}, \ldots, a_{k}, c_{1}, \ldots, c_{k} \in M$ and $\left(\mathscr{M}, a_{1}, \ldots, a_{k}\right) \equiv_{\Delta_{n+1}}\left(\mathscr{M}, c_{1}, \ldots, c_{k}\right)$. Let $a_{k+1}$ be any element of $M$. Then there exists a $c_{k+1} \in M$ such that

$$
\left(\mathscr{M}, a_{1}, \ldots, a_{k+1}\right) \equiv_{\Delta_{n}}\left(\mathscr{M}, c_{1}, \ldots, c_{k+1}\right) .
$$

Proof. The lemma is true but uninteresting if $\mathscr{M}$ is standard, so we will assume that $\mathscr{M}$ is nonstandard. Let $\tau\left(v, a_{1}, \ldots, a_{k+1}, c_{1}, \ldots, c_{k}\right)$ denote the following recursive set of $\Delta_{n}$-formulas:

$$
\begin{aligned}
\tau(v, \vec{a}, \vec{c})=\left\langle\varphi_{i}\left(a_{1}, \ldots, a_{k}, a_{k+1}\right)\right. & \leftrightarrow \varphi_{i}\left(c_{1}, \ldots, c_{k}, v\right): \\
\varphi_{i}\left(x_{1}, \ldots, x_{k+1}\right) & \text { is a parameter-free } \left.\Delta_{n} \text {-formula }\right\rangle .
\end{aligned}
$$


Then $\tau(v, \vec{a}, \vec{c})$ is a $\Delta_{n}$-type. For if we define $\varepsilon_{i}$ for each $i \in \omega$ by

$$
\varepsilon_{i}=\left\{\begin{aligned}
+1 & \text { if } \mathscr{M} \vDash \varphi_{i}\left(a_{1}, \ldots, a_{k+1}\right), \\
-1 & \text { if } \mathscr{M} \vDash \neg \varphi_{i}\left(a_{1}, \ldots, a_{k+1}\right),
\end{aligned}\right.
$$

then for any $l \in \omega$ we have

$$
\mathscr{M} \vDash \exists x \bigwedge_{i \leq l} \varepsilon_{i} \varphi_{i}\left(a_{1}, \ldots, a_{k}, x\right) .
$$

Here we take $(+1) \varphi$ to be $\varphi$ and $(-1) \varphi$ to be $\neg \varphi$. Note that this is a $\Sigma_{n}$ formula since $\varphi_{i}$ and $\neg \varphi_{i}$ are $\Delta_{n}$ for each $i$. (The assumption that $n \geq 1$ comes in here, as $\Sigma_{0}$ is not closed under existential quantification.) By the hypotheses of the lemma, then,

$$
\mathscr{M} \vDash \exists v \bigwedge_{i \leq l} \varepsilon_{i} \varphi_{i}\left(c_{1}, \ldots, c_{k}, v\right),
$$

and any $v$ which works in this formula also satisfies the first $l+1$ formulas of $\tau$.

By Lemma 3.5, $\tau$ is realized by some $c_{k+1} \in M$ which therefore satisfies the conclusion of the lemma.

The proof shows that we can replace $\Delta_{n+1}$ by $\Sigma_{n}$ in the hypothesis of the lemma, but the form given here is more convenient for proofs using induction on $n$.

Another property of recursively saturated models of PA is that they are tall. That is, if $\mathscr{M}$ is recursively saturated and $a_{1}, \ldots, a_{k} \in \mathscr{M}$, then the set of elements of $M$ definable using only the parameters $a_{1}, \ldots, a_{k}$ is not cofinal in $\mathscr{M}$ (see [Smo2]). The appropriate version of this result for arbitrary nonstandard models is:

Lemma 3.8. Let $\mathscr{M} \vDash \mathrm{PA}$ be nonstandard, $n \in \omega$, and $a_{1}, \ldots, a_{k} \in M$. Let $H_{\Sigma_{n}}\left(a_{1}, \ldots, a_{k}\right)$, the $\Sigma_{n}$-hull of $a_{1}, \ldots, a_{k}$, denote the set of elements of $M$ definable by $\Sigma_{n}$-formulas whose parameters are among $a_{1}, \ldots, a_{k}$. Then $H_{\Sigma_{n}}\left(a_{1}, \ldots, a_{k}\right)$ is not cofinal in $\mathscr{M}$.

Proof. The set of formulas

$$
\begin{aligned}
\tau=\left\langle v>\mu x \varphi_{i}\left(x, a_{1}, \ldots, a_{k}\right):\right. & \varphi_{i}\left(x, y_{1}, \ldots, y_{k}\right) \\
& \text { is a parameter-free } \left.\Sigma_{n} \text {-formula }\right\rangle
\end{aligned}
$$

is a recursive $\Pi_{n+1}$-type (once we rewrite the formulas without the $\mu$-operator), so it is realized. Any $b \in M$ realizing $\tau$ is bigger than all the elements of $H_{\Sigma_{n}}\left(a_{1}, \ldots, a_{k}\right)$.

Now we are in a position to show that if $\mathscr{M} \vDash \mathrm{PA}$ is nonstandard, then there are end extendible subsets of $M$ which are not in $\operatorname{Def}(\mathscr{M})$. We will in fact show that $\omega$ is such a subset. Let $L^{\prime}$ be $L$ together with a unary predicate symbol (also denoted by $\omega$ ) for $\omega$, and let $\mathscr{M} \vDash$ PA be nonstandard. In $L^{\prime}(\mathscr{M}, \omega)$ we can express many notions which cannot be expressed in $L(\mathscr{M})$. For example, we can say that two elements of $\mathscr{M}$ are in the same block (i.e. 
their difference is finite) or that they have the same standard prime factors. The sets in the standard system $\operatorname{SSy}(\mathscr{M})$ are also definable in $(\mathscr{M}, \omega)$ (cf. [Smo2]). We can also show:

Lemma 3.9. Let $\mathscr{M} \vDash \mathrm{PA}$ be nonstandard. There is a formula $\psi(x, y, z ; \omega)$ of $L^{\prime}(\mathscr{M}, \omega)$ such that if $a, b, c \in \mathscr{M}$ then

$$
(\mathscr{M}, \omega) \vDash \psi(a, c, b ; \omega) \Leftrightarrow a \text { and } c \text { have the same } \Sigma_{n} \text {-type over } b .
$$

(Similarly for $\Pi_{n}, \Delta_{n}$.)

Proof. The set of Gödel numbers of $\Sigma_{n}$-formulas of $L$ with two free variables is recursive, hence there is an $L(\mathscr{M})$-formula $\theta(u)$ such that for all ${ }^{\ulcorner} \varphi^{\urcorner} \in \omega$, $\mathscr{M} \vDash \theta\left(\left\ulcorner\varphi^{\urcorner}\right) \Leftrightarrow \varphi\right.$ is a $\Sigma_{n}$-formula of $L$ with two free variables. Let $\psi$ be the following $L^{\prime}(\mathscr{M})$-formula:

$$
\begin{aligned}
\psi(x, y, z ; \omega) \equiv \forall\ulcorner\varphi\urcorner & (\omega(\ulcorner\varphi\urcorner) \wedge \theta(\ulcorner\varphi\urcorner)) \\
& \left.\rightarrow\left(\operatorname{Sat}_{\Sigma_{n}}^{2}(\ulcorner\varphi\urcorner, x, z) \leftrightarrow \operatorname{Sat}_{\Sigma_{n}}^{2}(\ulcorner\varphi\urcorner, y, z)\right)\right] .
\end{aligned}
$$

The above is more than just another example. We will show that the preceding result in some sense characterizes the limits of the expressive power of $L^{\prime}(\mathscr{M}, \omega)$.

Theorem 3.10. Let $\mathscr{M} \vDash \mathrm{PA}, a, b, c \in \mathscr{M}$, and $n \in \omega$. If $a$ and $c$ have the same $\Delta_{n+1}$-type (with respect to $L$ ) over $b$, then $a$ and $c$ have the same $\left(\exists_{n} \cup \forall_{n}\right)$-type (with respect to $L^{\prime}$ ) over $b$.

Proof. For $\mathscr{M}=\mathbb{N}$ this is trivial since $\Delta_{n+1} \supseteq \exists_{n} \cup \forall_{n}$, so suppose $\mathscr{M}$ is nonstandard. Note that the pairing function $\beta$ and the corresponding projection functions $\pi_{1}$ and $\pi_{2}$ in $L$ enable us to encode and decode finite sequences without affecting quantifier complexity in $L^{\prime}$ (where our quantifier hierarchy does not provide for bounded quantification).

The proof is by induction on $n$. It is more convenient to perform the induction on the following equivalent statement of the theorem: Let $\varphi(x, y ; \omega)$ be a $\exists_{n}$ - or $\forall_{n}$-formula of $L^{\prime}$, and suppose $a, b, c \in M$ are such that $a$ and $c$ have the same $\Delta_{n+1}$-type over $b$ and $(\mathscr{M}, \omega) \vDash \varphi(a, b ; \omega)$. Then $(\mathscr{M}, \omega) \vDash \varphi(c, b ; \omega)$.

(i) Let $n=0$. Then $\varphi$ is a quantifier-free $L^{\prime}$-formula. We induct on the complexity of $\varphi$; in this case it suffices to assume that $a$ and $c$ have the same $\Delta_{0}$-type over $b$.

(a) Suppose $\varphi$ is atomic. If $\omega$ does not appear in $\varphi$ the theorem is obvious, so we can assume that $\varphi$ is of the form $\omega(t(x, b))$, where $t(x, b)$ is an $L$-term. Suppose $a \in M$ is such that $(\mathscr{M}, \omega) \vDash \omega(t(a, b))$. Then $t(a, b)=e$ for some $e \in \omega$, so the $\Delta_{0}$-type of $a$ over $b$ contains the formula

$$
t(x, b)=\underbrace{1+1+\cdots+1}_{e \text { times }} .
$$


Therefore if $c$ has the same $\Delta_{0}$-type over $b$ as $a$ does, then $t(c, b)=e$ as well, so $(\mathscr{M}, \omega) \vDash \omega(t(c, b))$.

(b) Let $\varphi$ be the negation of an atomic $L^{\prime}$-formula; this time we can assume that $\varphi$ is $\neg \omega(t(x, b))$ and that $(\mathscr{M}, \omega) \vDash \neg \omega(t(a, b))$. The $\Delta_{0}$-type of $a$ over $b$ therefore contains all formulas of the form

$$
\neg(t(x, b)=\underbrace{1+1+\cdots+1}_{e \text { times }})
$$

for all $e \in \omega$; when $e=0$ the intended meaning of the above is $\neg(t(x, b)=0)$. If $c$ has the same $\Delta_{0}$-type over $b$ as $a$ does, then from the above it is clear that $(\mathscr{M}, \omega) \vDash \neg \omega(t(c, b))$.

If (c) $\varphi=\psi \vee \theta$ or (d) $\varphi=\psi \wedge \theta$, the proof follows easily by induction.

Since any quantifier-free formula can be written in disjunctive normal form (cf. [CK]), this completes the proof for $n=0$.

(ii) Assume the theorem for $n$ and suppose that $\varphi$ is a $\exists_{n+1}$ - or $\forall_{n+1}$-formula of $L^{\prime}$. We consider the two cases in turn.

(a) If $\varphi$ is a $\exists_{n+1}$-formula of $L^{\prime}$, say

$$
\varphi(x, y ; \omega) \equiv \exists z \psi(z, x, y ; \omega),
$$

then the fact that $L$ contains symbols for pairing and projection functions enables us to assume that $\psi(z, x, y ; \omega)$ is a $\forall_{n}$-formula of $L^{\prime}$. Let $a, b \in M$ be such that

$$
(\mathscr{M}, \omega) \vDash \exists z \psi(z, a, b ; \omega),
$$

and let $d \in M$ be such that

$$
(\mathscr{M}, \omega) \vDash \psi(d, a, b ; \omega) .
$$

Suppose $c$ and $a$ have the same $\Delta_{n+2}$-type over $b$. By Lemma 3.7, there is an $e \in M$ such that

$$
(\mathscr{M}, a, b, d) \equiv_{\Delta_{n+1}}(\mathscr{M}, c, b, e) \text {. }
$$

By the induction hypothesis, then,

$$
(\mathscr{M}, \omega) \vDash \psi(e, c, b ; \omega) .
$$

(Actually this requires a short detour in our argument. Define $\psi^{\prime}(u, y ; \omega)$ to be $\psi\left(\pi_{1}(u), \pi_{2}(u), y ; \omega\right)$. Then $\psi^{\prime}$ is a $\forall_{n}$-formula of $L^{\prime}$ and $(\mathscr{M}, \omega) \vDash$ $\psi^{\prime}(\beta(d, a), b ; \omega)$. Now $\beta(e, c)$ has the same $\Delta_{n+1}$-type over $b$ as $\beta(d, a)$ does, so the induction hypothesis tells us that $(\mathscr{M}, \omega) \vDash \psi^{\prime}(\beta(e, c), b ; \omega)$. In other words $(\mathscr{M}, \omega) \vDash \psi(e, c, b ; \omega)$.)

Therefore $(\mathscr{M}, \omega) \vDash \exists z \psi(z, c, b ; \omega)$.

(b) If $\varphi$ is an $\forall_{n+1}$-formula of $L^{\prime}$, say

$$
\varphi(x, y ; \omega) \equiv \forall z \psi(z, x, y ; \omega),
$$

we can assume that $\psi(z, x, y ; \omega)$ is a $\exists_{n}$-formula of $L^{\prime}$. Let $a, b \in M$ be such that

$$
(\mathscr{M}, \omega) \vDash \forall z \psi(z, a, b ; \omega) .
$$


Suppose $c$ and $a$ have the same $\Delta_{n+2}$-type over $b$, and let $e \in M$ be arbitrary. Lemma 3.7 gives us a $d \in M$ such that

$$
(\mathscr{M}, c, b, e) \equiv_{\Delta_{n+1}}(\mathscr{M}, a, b, d) \text {. }
$$

Now $(*)$ implies in particular that

$$
(\mathscr{M}, \omega) \vDash \psi(d, a, b ; \omega) .
$$

By the induction hypothesis (as in part (a)),

$$
(\mathscr{M}, \omega) \vDash \psi(e, c, b ; \omega) .
$$

But $e \in M$ was arbitrary, therefore

$$
(\mathscr{M}, \omega) \vDash \forall z \psi(z, c, b ; \omega)
$$

which completes the induction and the proof.

We can now prove:

Theorem 3.11. Let $\mathscr{M} \vDash$ PA be nonstandard. Then $\omega \subseteq M$ is uniquely end extendible.

Proof. Uniqueness is obvious. To show that $\omega$ is end extendible, suppose $\mathscr{M} \prec_{e} \mathscr{N}$ and let $\varphi(x, y ; \omega)$ be an $L^{\prime}$-formula and $b \in M$ be such that

$$
(\mathcal{N}, \omega) \vDash \exists x \varphi(x, b ; \omega) .
$$

To show that $(\mathscr{M}, \omega) \prec_{e}(\mathscr{N}, \omega)$ it suffices by Proposition 1.1 (and the fact that $L$ contains $\beta, \pi_{1}$, and $\pi_{2}$ ) to show that in the above situation we can always find a $c \in M$ such that

$$
(\mathcal{N}, \omega) \vDash \varphi(c, b ; \omega) .
$$

Take $n$ such that $\varphi(x, y ; \omega) \in \exists_{n} \cup \forall_{n}$. Also let $a \in N$ be such that $(\mathcal{N}, \omega) \vDash \varphi(a, b ; \omega)$. By Theorem 3.10, any $c \in N$ with the same $\Delta_{n+1}$-type as $a$ (with respect to $L$ ) over $b$ will also satisfy $\varphi$ in $(\mathscr{N}, \omega)$.

Now $b \in M$, so by Lemma 3.8 we can find an $m \in M$ such that $m$ is greater than everything in $H_{\Delta_{n+1}}^{\mathscr{K}}(b)$. But $H_{\Delta_{n+1}}^{\mathcal{U}}(b)=H_{\Delta_{n+1}}^{\text {II }}(b)$, therefore the following set of $L(\mathcal{N})$-formulas is a $\Delta_{n+1}$-type in $L(\mathcal{N})$ over $a, b, m$ :

$$
\begin{aligned}
\tau(v, a, b, m) \equiv\{v<m\} \cup & \left\langle\psi_{i}(v, b) \leftrightarrow \psi_{i}(a, b):\right. \\
& \left.\psi_{i}(x, y) \text { is a parameter-free } \Delta_{n+1} \text {-formula of } L\right\rangle .
\end{aligned}
$$

By Lemma 3.5, $\tau$ is realized by some $c \in N$. But $c<m \in M$ and $\mathscr{M} \prec_{e} \mathscr{N}$, therefore $c \in M$. Clearly $c$ has the same $\Delta_{n+1}$-type as $a$ over $b$.

Note that we can replace $\omega$ by an arbitrary subset of $\omega$ in Theorems 3.10 and 3.11; these results and their proofs go over basically unchanged. Our next objective is more ambitious: We want to replace $\omega$ by an arbitrary bounded subset of $M$ in Theorem 3.11. We could have proven the resulting generalization directly, but the proof is more difficult and Theorem 3.10 is interesting in its own right. Furthermore, in Theorems 3.10 and 3.11 we never used bounded 
quantifiers-we could replace $\Delta_{n+1}$ by $D_{n+1}$ in Theorem 3.10, which implies that we can generalize to languages for which $\Sigma_{n}$ and $\exists_{n}$ are not equivalent. On the other hand, we will make essential use of bounded quantification in what follows.

Let $\mathscr{M} \vDash$ PA be nonstandard and let $S \subseteq M$ be a bounded subset of the universe; suppose $S<m \in M$. To show that $S$ is end extendible we will need some sort of analogue to Theorem 3.10. The first problem we face is how to handle atomic and negated atomic formulas, since $S$ (unlike $\omega$ ) will not be pointwise definable in general. Our solution is to required that $a$ and $c$ have the same $\Delta_{n+1}$-type over $b, r$ for all $r<m$, and we utilize bounded quantifiers in order to accomplish this.

In order to handle the induction step, we need to generalize Lemma 3.7.

Lemma 3.12. Let $\mathscr{M} \vDash \mathrm{PA}, n \geq 1$, and suppose $a_{1}, \ldots, a_{k}, c_{1}, \ldots, c_{k}, m \in M$ and $\left(\mathscr{M}, a_{1}, \ldots, a_{k}, r\right) \equiv_{\Delta_{n+1}}\left(\mathscr{M}, c_{1}, \ldots, c_{k}, r\right)$ for all $r<2^{2^{m}}$. Let $a_{k+1}$ be any element of $M$. Then there exists a $c_{k+1} \in M$ such that

$$
\left(\mathscr{M}_{1} a_{1}, \ldots, a_{k+1}, r\right) \equiv_{\Delta_{n}}\left(\mathscr{M}_{1} c_{1}, \ldots, c_{k+1}, r\right)
$$

for all $r<m$.

Proof. Again we can assume that $\mathscr{M}$ is nonstandard. Let $\tau$ denote the following recursive set of $\Delta_{n}$-formulas:

$$
\begin{aligned}
\tau \equiv\left\langle\forall u<m\left(\varphi_{i}\left(a_{1}, \ldots, a_{k}, a_{k+1}, u\right) \leftrightarrow \varphi_{i}\left(c_{1}, \ldots, c_{k}, v, u\right)\right):\right. \\
\left.\varphi\left(x_{1}, \ldots, x_{k+1}, u\right) \text { is a parameter-free } \Delta_{n} \text {-formula }\right\rangle .
\end{aligned}
$$

We will show that $\tau(v, \vec{a}, \vec{c})$ is a type.

Let $l \in \omega$; we must show that

$$
\mathscr{M} \vDash \exists v \forall u<m \bigwedge_{i \leq l}\left(\varphi_{i}\left(a_{1}, \ldots, a_{k+1}, u\right) \leftrightarrow \varphi_{i}\left(c_{1}, \ldots, c_{k}, v, u\right)\right) .
$$

Recall the canonical indexing of finite sets; the $y$ th finite set $D_{y}$ is given by

$$
\begin{gathered}
D_{y}=\varnothing \text { if } y=0, \\
D_{y}=\left\{y_{0}, \ldots, y_{t-1}\right\} \quad \text { if } y=2^{y_{0}}+2^{y_{1}}+\cdots+2^{y_{t-1}} \\
\text { and } y_{0}<y_{1}<\cdots<y_{t-1}
\end{gathered}
$$

(see [Smo2]). The internal cardinality $t$ of $D_{y}$ is denoted by $\left|D_{y}\right|$. Note that the formula $y_{j} \in D_{y}$ is provably $\Delta_{1}$. Note also that if $D_{e}$ is an $\mathscr{M}$-finite set all of whose members are less than some $m^{\prime} \in M$, then $e<2^{m^{\prime}}$ in $\mathscr{M}$.

For each $i \leq l$, the set

$$
\left\{u<m: \mathscr{M} \vDash \varphi_{i}\left(a_{1}, \ldots, a_{k+1}, u\right)\right\}
$$

is $\mathscr{M}$-finite and its elements are all less than $m$, hence by Arithmetic Separation it is coded by some $r_{i}<2^{m}$. Let $D_{r}$ be the (genuinely) finite set containing all 
the $r_{i}$ 's for $i \leq l$; then $\operatorname{card}\left(D_{r}\right) \leq l+1$ since the $r_{i}$ might not all be distinct, and $r<2^{2^{m}}$.

By assumption $a_{1}, \ldots, a_{k}$ and $c_{1}, \ldots, c_{k}$ have the same $\Delta_{n+1}$-type over $r$, hence over $r_{0}, \ldots, r_{l}$ which are $\Delta_{1}$-definable from $r$. For each $i \leq l$, the definition of $r_{i}$ tells us that

$$
\mathscr{M} \vDash \forall u<m\left(\varphi_{i}\left(a_{1}, \ldots, a_{k+1}, u\right) \leftrightarrow u \in D_{r_{i}}\right) .
$$

Therefore we have

$$
\mathscr{M} \vDash \exists v \forall u<m \bigwedge_{i \leq l}\left(\varphi_{i}\left(a_{1}, \ldots, a_{k}, v, u\right) \leftrightarrow u \in D_{r_{i}}\right) .
$$

Since $u \in D_{r_{i}}$ is $\Delta_{1}$, and $n \geq 1$, this formula is $\Delta_{n+1}$. So

$$
\mathscr{M} \vDash \exists v \forall u<m \bigwedge_{i \leq l}\left(\varphi_{i}\left(c_{1}, \ldots, c_{k}, v, u\right) \leftrightarrow u \in D_{r_{i}}\right) .
$$

Any $v$ satisfying the above also satisfies the first $l+1$ formulas of $\tau$. By Lemma $3.5, \tau$ is realized by some $c_{k+1} \in M$ which therefore satisfies the conclusion of the lemma.

We have since discovered the nonhierarchical version of the above lemma for recursively saturated models in C. Smorynski's paper [Smo3], with a similar proof. We are now in a position to generalize Theorem 3.10.

Theorem 3.13. Let $\mathscr{M} \vDash$ PA and let $S \subseteq M$ be a bounded subset of $M$. Let $L_{S}$ be the language obtained by adjoining a unary predicate (also denoted by $S$ ) for $S$ to $L$. Fix $m \in M$ such that $S<m$. Define $g_{j}(m)$ by induction on $j$ such that $g_{0}(m)=m$ and $g_{j+1}(m)=2^{2^{g_{j}(m)}}$.

Let $a, b, c \in M$ and $n \in \omega$. If $a$ and $c$ have the same $\Delta_{n+1}$-type (with respect to $L$ ) over $b, r$ for all $r<g_{n}(m)$, then $a$ and $c$ have the same $\left(\exists_{n} \cup \forall_{n}\right)$-type (with respect to $L_{S}$ ) over $b$.

Proof. Again we can assume that $\mathscr{M}$ is nonstandard, and our other observations at the beginning of the proof of Theorem 3.10 apply here as well. Again the proof is by induction on $n$; this time we restate the theorem as follows: Let $\varphi(x, y ; S)$ be a $\exists_{n}$ - or $\forall_{n}$-formula of $L_{S}$, and suppose $a, b, c \in M$ are such that $a$ and $c$ have the same $\Delta_{n+1}$-type over $b, r$ for all $r<g_{n}(m)$, and that $(\mathscr{M}, S) \vDash \varphi(a, b ; S)$. Then $(\mathscr{M}, S) \vDash \varphi(c, b ; S)$.

(i) Let $n=0$. Then $\varphi$ is a quantifier-free $L_{S}$-formula. We induct on the complexity of $\varphi$; it suffices in this case to assume that $a$ and $c$ have the same $\Delta_{0}$-type over $b, r$ for all $r<m$.

(a) Suppose $\varphi$ is atomic; we can assume that $\varphi$ is of the form $S(t(x, b))$, where $t(x, b)$ is an $L$-term. Let $a \in M$ be such that $(\mathscr{M}, S) \vDash S(t(a, b))$. Then $t(a, b)=s$ for some $s \in S$, so the $\Delta_{0}$-type of $a$ over $b$ and $s$ contains the formula $t(x, b)=s$. But $s<m$, so if $c \in M$ has the same $\Delta_{0}$-type as $a$ over $b$ and all $r$ such that $r<m$, then in particular the $\Delta_{0}$-type of $c$ over $b$ and $s$ contains the formula $t(x, b)=s$. Therefore $(\mathscr{M}, S) \vDash S(t(c, b))$. 
(b) Let $\varphi$ be the negation of an atomic $L_{S}$-formula; assume $\varphi$ is $\neg S(t(x, b))$ and that $(\mathscr{M}, S) \vDash \neg S(t(a, b))$. Then for each $s \in S$, the $\Delta_{0}$-type of $a$ over $b, s$ contains the formula $\neg t(x, b)=s$. By assumption, for each $s \in S$ the $\Delta_{0}$-type of $c$ over $b, s$ also contains the formula $\neg t(x, b)=s$, from which we can conclude that $(\mathscr{M}, S) \vDash \neg S(t(c, b))$.

If (c) $\varphi=\psi \vee \theta$ or (d) $\varphi=\psi \wedge \theta$, the proof follows easily by induction.

Any quantifier-free formula can be written in disjunctive normal form, hence this completes the proof for $n=0$.

(ii) Assume the theorem for $n$ and suppose that $\varphi$ is a $\exists_{n+1}$ - or $\forall_{n+1}$-formula of $L_{S}$.

(a) Suppose $\varphi(x, y ; S) \equiv \exists z \psi(z, x, y ; S)$ is a $\exists_{n+1}$-formula of $L_{S}$, where $\psi$ is a $\forall_{n}$-formula. Let $a, b \in M$ be such that

$$
(\mathscr{M}, S) \vDash \exists z \psi(z, a, b ; S) .
$$

Let $d \in M$ be such that

$$
(\mathscr{M}, S) \vDash \psi(d, a, b ; S) .
$$

Suppose $c$ and $a$ have the same $\Delta_{n+2}$-type (with respect to $L$ ) over $b, r$ for all $r<g_{n+1}(m)$. By Lemma 3.12, there is an $e \in M$ such that

$$
(\mathscr{M}, a, b, d, r) \equiv_{\Delta_{n+1}}(\mathscr{M}, c, b, e, r)
$$

for all $r<g_{n}(m)$. By the induction hypothesis,

$$
(\mathscr{M}, S) \vDash \psi(e, c, b ; S) \text {. }
$$

(See the remark which appears at the corresponding point in the proof of Theorem 3.10.) Thus

$$
(\mathscr{M}, S) \vDash \exists z \psi(z, c, b ; S) .
$$

(b) Let $\varphi(x, y ; S) \equiv \forall z \psi(z, x, y ; S)$ be a $\forall_{n+1}$-formula of $L_{S}$, where $\psi$ is a $\exists_{n}$-formula. Let $a, b \in M$ be such that

$$
(\mathscr{M}, S) \vDash \forall z \psi(z, a, b ; S) .
$$

Suppose $c$ and $a$ have the same $\Delta_{n+2}$-type over $b, r$ for all $r<g_{n+1}(m)$, and let $e \in M$ be arbitrary. By Lemma 3.12 we can find a $d \in M$ such that

$$
(\mathscr{M}, c, b, e, r) \equiv_{\Delta_{n+1}}(\mathscr{M}, a, b, d, r)
$$

for all $r<g_{n}(m)$.

Now $(*)$ implies in particular that

$$
(\mathscr{M}, S) \vDash \psi(d, a, b ; S) .
$$

By the induction hypothesis,

$$
(\mathscr{M}, S) \vDash \psi(e, c, b ; S) .
$$

But $e \in M$ was arbitrary, therefore

$$
(\mathscr{U}, S) \vDash \forall z \psi(z, c, b ; S),
$$

which completes the induction and the proof.

We now prove the main result in this section. 
Theorem 3.14. Let $\mathscr{M} \vDash \mathrm{PA}$ and let $S \subseteq M$ be a bounded subset of $M$. Then $S$ is uniquely end extendible.

Proof. Uniqueness is obvious, as is the theorem for $\mathscr{M}=\mathbb{N}$. Let $\mathscr{M}$ be nonstandard and $S \subseteq M$ be bounded by $m \in M$. Suppose $\mathscr{M} \prec_{e} \mathscr{N}, \varphi(x, y ; S)$ is an $L_{S}$-formula, and $b \in M$ is such that

$$
(\mathscr{N}, S) \vDash \exists x \varphi(x, b ; S) .
$$

To show that $(\mathscr{M}, S) \prec_{e}(\mathscr{N}, S)$, it suffices to show that in the above situation we can find a $c \in M$ such that

$$
(\mathcal{N}, S) \vDash \varphi(c, b ; S)
$$

Take $n$ such that $\varphi(x, y ; S) \in \exists_{n} \cup \forall_{n}$. Let $a \in N$ be such that $(\mathscr{N}, S)$ F $\varphi(a, b ; S)$. By Theorem 3.13, any $c \in N$ with the same $\Delta_{n+1}$-type as $a$ over $b, r$ for all $r<g_{n}(m)$ will also satisfy $\varphi(x, b ; S)$ in $(\mathcal{N}, S)$.

Now $b \in M$ and $n \in M$. By Lemma 3.8, there is an $m^{\prime} \in M$ such that $m^{\prime}$ is greater than everything in $H_{\Delta_{n+1}}^{\mathscr{K}}(b, m)=H_{\Delta_{n+1}}^{\mathcal{N}}(b, m)$. Consider the following set of $\Delta_{n+1}$-formulas of $L(M)$ :

$$
\begin{aligned}
& \tau\left(v, a, b, m, m^{\prime}\right) \equiv\left\{v<m^{\prime}\right\} \\
& \qquad\left\langle\forall u<g_{n}(m)\left[\psi_{i}(v, b, u) \leftrightarrow \psi_{i}(a, b, u)\right]:\right. \\
& \left.\quad \psi_{i}(x, y, u) \text { is a parameter-free } \Delta_{n+1} \text {-formula of } L\right\rangle .
\end{aligned}
$$

If we examine the proof of Lemma 3.12, we see that for any $l \in \omega$ there is an $r<g_{n+1}(m)$ such that

$$
\mathscr{M} \vDash \exists v \forall u<g_{n}(m) \bigwedge_{i \leq l}\left(\psi_{i}(v, b, u) \leftrightarrow u \in D_{r_{i}}\right)
$$

(where the $r_{i}$ 's are definable from $r$ as in the aforementioned proof), and such that any $v$ which instantiates this statement also satisfies the first $l+1$ formulas in the second clause of $\tau$. The least such $v$ belongs to $H_{\Delta_{n+1}}^{\mathscr{M}}(b, m, r)$. But $r<g_{n+1}(m)$, so it is easy to prove that no element of $H_{\Delta_{n+1}}^{\mathscr{⿲}}(b, m, r)=$ $H_{\Delta_{n+1}}^{\prime \prime}(b, m, r)$ is bigger than everything in $H_{\Delta_{n+1}}(b, m)$. Thus we can find such a $v$ which is less than $m^{\prime}$, so $\tau$ is a $\Delta_{n+1}$-type in $L(\mathscr{M})$.

By Lemma 3.5, $\tau$ is realized by some $c \in N$. But $c<m^{\prime} \in M$, hence $c \in M$. Clearly $c$ has the same $\Delta_{n+1}$-type as $a$ over $b, r$ for all $r<g_{n}(m)$.

A special case of the above theorem (for $\mathscr{M}, \mathscr{N}$ countable recursively saturated models and $S$ an initial segment of $\mathscr{M}$ ) appears in [Smo3], along with the result that under these assumptions we also have $(\mathscr{M}, S) \cong(\mathscr{N}, S)$ by an isomorphism which is the identity on $S$.

Combining Theorems 3.3 and 3.14 and using Proposition 2.1(ii), we can conclude the following. 
Corollary 3.15. Let $\mathscr{M} \vDash$ PA be nonstandard, $C$ a proper class in $\mathscr{M}$, and $S$ a bounded subset of $M$. Then $C \notin \operatorname{Def}(\mathscr{M}, S)$.

Our characterization of the set of end extendible subsets of $\mathscr{M}$ is still incomplete (except when $\mathscr{M}=\mathbb{N}$ ); we are left with the case when $U \subseteq M$ is undefinable and unbounded in $\mathscr{M}$ and is such that no proper class $C$ of $\mathscr{M}$ is in $\operatorname{Def}(\mathscr{M}, U)$.

We close this section with the following result, which applies in particular to all countable nonstandard $\mathscr{M}$.

Proposition 3.16. Let $\mathscr{M} \vDash \mathrm{PA}$ be nonstandard of cardinality $\kappa$ and suppose $\mathscr{M}$ is not $\kappa$-like. Then there is a subset $U \subseteq M$ which is not weakly end extendible. Proof. By assumption there exists an $a \in M$ such that $[0, a]$ has cardinality $\kappa$. Let $g:[0, a] \rightarrow M$ be any function whose image is unbounded in $M$, and let $U=\{\beta(m, g(m)): m \leq a\}$. Clearly $U$ is not weakly end extendible.

In contrast to the above, if $\mathscr{M}$ is any model of PA and $U$ is an arbitrary inductive subset of $M$, we can apply the MacDowell-Specker Theorem to $(\mathscr{M}, U)$ and conclude that $U$ is weakly end-extendible.

\section{Cofinal extendibility}

This section is the counterpart of $\S 3$ for cofinal extensions. We begin with the obvious:

Definition 4.1. Let $\mathscr{M} \vDash \mathrm{PA}, U \subseteq M . U$ is cofinally extendible if $U$ is extendible to $\mathscr{N}$ whenever $\mathscr{M} \prec_{c} \mathscr{N}$.

For most of this section we will need to assume $\mathscr{M}$ is countable. Our reward for making this restriction is Theorem 4.15 , which completely characterizes the cofinally extendible subsets of any countable nonstandard $\mathscr{M} \vDash$ PA.

Before we invoke our countability assumption we mention a couple of things about the general case. First of all, the existence of proper cofinal extensions of arbitrary nonstandard $\mathscr{M}$ is a consequence of the Elementary Splitting Theorem (Theorem 0.4). Secondly, one direction of our Theorem 4.15 is due to J. Schmerl and $\mathrm{H}$. Kotlarski (independently) and requires no assumptions about the cardinality of $\mathscr{M}$ :

Theorem 4.2. Let $\mathscr{M} \vDash \mathrm{PA}$ be nonstandard and suppose $U \subseteq M$ is inductive. Then $U$ is uniquely cofinally extendible.

As proofs of this theorem appear both in [Ko] and [Sch], and the theorem in any case is a generalization of Gaifman's Cofinality Theorem in $[G]$, we content ourselves with the observation that the uniqueness is obvious. For suppose $\mathscr{M} \prec_{c} \mathscr{N}$ and $V \subseteq N$ is such that $(\mathscr{M}, U) \prec(\mathscr{N}, V) . U$ is a class, so for every $a \in M,\left\{u \in U: u<^{\mathscr{\prime}} a\right\}$ is an $\mathscr{M}$-finite set. Say $b \in M$ is such that

$$
(\mathscr{M}, U) \vDash \forall u<a\left(U(u) \leftrightarrow u \in D_{b}\right) .
$$

Then $(\mathscr{N}, V) \vDash \forall u<a\left(V(u) \leftrightarrow u \in D_{b}\right)$. 
Since $\mathscr{M}$ is cofinal in $\mathscr{N}$, all the initial segments of $V$ are determined by the above, so $V$ itself is uniquely determined.

Compare the above result with Theorem 3.3. To complete the proof of Theorem 0.3 , we will prove the converse to Theorem 4.2 for countable $\mathscr{M}$.

For the remainder of this section, we assume that the model $\mathscr{M}$ is countable and nonstandard.

Let $U \subseteq M$ be a subset of $M$ which is not inductive. Then $\operatorname{Def}(\mathscr{M}, U)$ contains a nonempty subset $W$ which does not have a least element. Let

$$
I=\left\{a \in M: \forall w \in W a<^{\mathscr{M}} w\right\} .
$$

Then $I$ is a cut of $\mathscr{M}$ and $I \in \operatorname{Def}(\mathscr{M}, U)$. If we prove that $I$ is not cofinally extendible, we can apply Proposition 2.1 to conclude that $U$ is not cofinally extendible either.

It is convenient at this point to introduce the following definition due to $\mathrm{L}$. Kirby [Ki].

Definition 4.3. Let $J$ be a cut of $\mathscr{M}$. Then $\operatorname{cf}^{\mathscr{M}}(J)$, the cofinality of $J$ in $\mathscr{M}$, is the set of $e \in M$ such that whenever $D_{s}$ has internal cardinality $\leq e$ (i.e. whenever $s \in M$ is such that $\left.\mathscr{M} \vDash\left|D_{s}\right| \leq e\right)$ then $D_{s} \cap J$ is not cofinal in $J$.

Lemma 4.4. Let $J$ be a cut of $\mathscr{M}$. Then $\mathrm{cf}^{\mathscr{\prime l}}(J)$ is also a cut of $\mathscr{M}, \operatorname{cf}^{\mathscr{K}}(J) \subseteq$ $J$, and $\operatorname{cf}^{\mathscr{\prime}}(J) \in \operatorname{Def}(\mathscr{M}, J)$.

We need one more definition and lemma, this time from Kaufmann and Schmerl [KS]. See $\S 1$ for terminology.

Definition 4.5. Let $e, i$, and $j$ be elements of the model $\mathscr{M}$ and suppose $C \in \operatorname{Def}(\mathscr{M})$. We think of $C$ as consisting of codes of sequences of length $e$. $C$ is $(i, j)$-rich (for $\mathscr{M}$ and $e$ ) if $j \geq 1$ and for every $d \in M$, there is a $c \in C$ such that

$$
(d)_{k}=(c)_{i+k j} \quad \text { whenever } i+k j<e .
$$

Lemma 4.6. Suppose that $C$ is $(i, j)$-rich for $\mathscr{M}$ and $e$, and that $f: C \rightarrow$ $[0, a)$ is parametrically definable in $\mathscr{M}$. Then for some $b<a, f^{-1}(\{b\})$ is $(i+b j, a j)$-rich (for $\mathscr{M}$ and $e$ ).

$\operatorname{Proof}([\mathrm{KS}])$. Suppose that for all $b<a, f^{-1}(\{b\})$ is not $(i+b j, a j)$-rich. For each $b<a$ let $d_{b}$ be least such that there is no $c \in f^{-1}(\{b\})$ satisfying

$$
\left(d_{b}\right)_{k}=(c)_{i+b j+k a j} \text { whenever } i+b j+k a j<e .
$$

We can code the sequence $\left\langle d_{b}: b<a\right\rangle$; let $d \in M$ be such that $(d)_{b+k a}=\left(d_{b}\right)_{k}$ whenever $i+b j+k a j<e$ and $b<a$.

Now $C$ is $(i, j)$-rich, hence there is a $c \in C$ such that $(d)_{b+k a}=(c)_{i+(b+k a) j}$ whenever $i+(b+k a) j<e$. In other words,

$$
\left(d_{b}\right)_{k}=(c)_{i+b j+k a j} \text { whenever } b<a \text { and } i+b j+k a j<e .
$$

Setting $b=f(c)$ gives us a contradiction.

Now we can prove that certain cuts of $\mathscr{M}$ are not cofinally extendible. 
Lemma 4.7. Let $I$ be a cut of $\mathscr{M}$ such that $\mathrm{cf}^{\mathscr{\prime}}(I) \neq \omega$. Then $I$ is not cofinally extendible.

Proof. Our strategy is to construct an elementary cofinal extension $\mathscr{N}$ of $\mathscr{M}$ such that $\mathcal{N}$ does not fill $I$ and $\operatorname{cf}^{\mathcal{N}}\left(I^{\mathcal{H}}\right)=\omega$. Here $I^{\mathcal{N}}$ denotes the cut of $\mathcal{N}$ determined by $I$ :

$$
I^{\mathcal{H}}=\left\{b \in N: \exists i \in I\left(b<<^{\iota} i\right)\right\} .
$$

Since $\mathscr{N}$ does not fill $I, I^{+}$is the only candidate for a set $J \subseteq N$ such that $(\mathscr{M}, I) \prec(\mathscr{N}, J)$. But by assumption there is a nonstandard $m \in M$ such that $m \in \operatorname{cf}^{\mathscr{M}}(I)$, whereas $m \notin \operatorname{cf}^{\mathscr{\prime \prime}}\left(I^{\prime \prime}\right)$. By Lemma 4.4, $\left(\mathscr{M}, I^{\mathscr{K}}\right) \nprec\left(\mathscr{N}, I^{\mathcal{H}}\right)$. Therefore $I$ is not cofinally extendible.

It remains to construct $\mathscr{N}$. We adapt the proof of Lemma 4.10 of [KS]. We will define a descending sequence of parametrically definable sets $C_{n} \quad(n \in \omega)$ with the property that for every $L(\mathscr{M})$-formula $\varphi(x)$ there is an $n \in \omega$ such that either $\varphi(c)$ holds for all $c \in C_{n}$ or else $\neg \varphi(c)$ holds for all $c \in C_{n}$. The sets $C_{n}$ thus determine a complete type over $\mathscr{M}$; if $b$ realizes this type, we can form the simple elementary extension $\mathscr{M}(b)$ of $\mathscr{M}$. By Theorem 0.4 there is a unique model $\mathscr{N}$ (which is the model we want) such that $\mathscr{M} \prec_{c} \mathscr{N} \prec_{e} \mathscr{M}(b)$.

We define the sequence $\left\langle C_{n}: n \in \omega\right\rangle$ by induction. Let $\left\langle a_{n}: n \in \omega\right\rangle$ be a sequence of elements of $I$ which is cofinal in $I$. Let $\left\langle\varphi_{n}(x): n \in \omega\right\rangle$ enumerate all $L(\mathscr{M})$-formulas with one free variable and let $\left\langle\psi_{n}(x, y): n \in \omega\right\rangle$ enumerate all $L(\mathscr{M})$-formulas with two free variables. Fix $e \in \mathscr{M} \backslash \omega$.

Set $C_{0}=M$, which is $(0,1)$-rich for $\mathscr{M}$ and $e$. Our inductive hypothesis is that $C_{n}$ is $(i, j)$-rich for $\mathscr{M}$ and $e$, where $i, j \in \omega$ are dependent on $n$.

We define $C_{n+1}$ from $C_{n}$ in stages. First let $C_{n}^{0}=\left\{c \in C_{n}:(c)_{i}=a_{n}\right\}$. Then $C_{n}^{0}$ is $\left(i_{0}, j_{0}\right)$-rich where $i_{0}=i+j \in \omega$ and $j_{0}=j \in \omega$.

Second, let $f: C_{n}^{0} \rightarrow[0,2)$ be defined by $f(c)=1 \Leftrightarrow \mathscr{M} \vDash \varphi_{n}(c)$ and $f(c)=0 \Leftrightarrow \mathscr{M} \vdash \neg \varphi_{n}(c)$. By Lemma 4.6, either $f^{-1}(\{0\})$ is $\left(i_{0}, 2 j_{0}\right)$-rich for $\mathscr{M}$ and $e$ or else $f^{-1}(\{1\})$ is $\left(i_{0}+j_{0}, 2 j_{0}\right)$-rich for $\mathscr{M}$ and $e$. Thus there exist $i_{1}, j_{1} \in \omega$ and $C_{n}^{1} \subseteq C_{n}^{0}$ such that $C_{n}^{1}$ is $\left(i_{1}, j_{1}\right)$-rich and either $\mathscr{M} \vDash \varphi_{n}(c)$ for all $c \in C_{n}^{1}$ or else $\mathscr{M} \vDash \neg \varphi_{n}(c)$ for all $c \in C_{n}^{1}$.

Finally we must ensure that $I$ is not filled in the extension. Let $h$ be least such that $\left\{c \in C_{n}^{1}: \mu y \psi_{n}(c, y) \leq h\right\}$ is $\left(i_{1}, 2 j_{1}\right)$-rich. (If no such $h$ exists, choose $h \in M \backslash I$ arbitrarily.)

If $h \in I$, set $C_{n+1}=\left\{c \in C_{n}^{1}: \mu y \psi_{n}(c, y) \leq h\right\}$. By assumption, then, $C_{n+1}$ is $\left(i_{1}, 2 j_{1}\right)$-rich in this case.

If $h \notin I$, set $C_{n+1}=\left\{c \in C_{n}^{1}: \mu y \psi_{n}(c, y) \geq h\right\}$. Define $f: C_{n}^{1} \rightarrow[0,2)$ by $f(c)=1 \Leftrightarrow \mu y \psi_{n}(c, y) \geq h$ and $f(c)=0 \Leftrightarrow \mu y \psi_{n}(c, y) \leq h-1$. By assumption $f^{-1}(\{0\})$ is not $\left(i_{1}, 2 j_{1}\right)$-rich, hence by Lemma 4.6 $C_{n+2}=f^{-1}(\{1\})$ is $\left(i_{1}+j_{1}, 2 j_{1}\right)$-rich.

This completes the construction of $\left\langle C_{n}: n \in \omega\right\rangle$. 
Let $\Sigma(v)=\left\{\varphi_{n}(v): \mathscr{M} \vDash \varphi_{n}(c)\right.$ for all $\left.c \in C_{n+1}\right\}$. Then $\Sigma(v)$ is a complete type over $\mathscr{M}$, so we can find a simple elementary extension $\mathscr{M}(b)$ of $\mathscr{M}$ such that $b$ realizes $\Sigma(v)$. Now $\mathscr{M}(b)$ does not fill $I$, as every element of $\mathscr{M}(b)$ is of the form $\mu y \psi_{n}(b, y)$ for some $n \in \omega$, and the construction ensures that either

$$
\mathscr{M}(b) \vDash \mu y \psi_{n}(b, y) \leq h
$$

for some $h \in I$, or else that

$$
\mathscr{M}(b) \vDash \mu y \psi_{n}(b, y) \geq h
$$

for some $h \in M \backslash I$.

Also by construction, for every $n \in \omega$ there is an $i \in \omega$ such that $\mathscr{M}(b) \vDash$ $(b)_{i}=a_{n}$. Thus for any nonstandard $d \in|\mathscr{M}(b)|$, the sequence $\left\langle(b)_{i}: i<d\right\rangle$ gives us an $\mathscr{M}(b)$-finite set of internal cardinality $\leq d$ whose intersection with $I$ is cofinal in $I$. Hence $\operatorname{cf}^{\mathscr{M}(b)}\left(I^{\mathscr{M}(b)}\right)=\omega$.

Finally, we can split the extension to find an $\mathscr{N}$ such that $\mathscr{M} \prec_{c} \mathscr{N} \prec_{e}$ $\mathscr{M}(b)$. Clearly $\mathscr{N}$ does not fill $I$, and for any nonstandard $d \in N$ and $d^{\prime} \in N \backslash I$ the set $\left\{(b)_{i}: i<d\right\} \cap\left[0, d^{\prime}\right)$ is $\mathscr{N}$-finite of internal cardinality $\leq d$ and its intersection with $I^{\mathcal{N}}$ is cofinal in $I^{\mathscr{N}}$. Thus $\mathrm{cf}^{\mathscr{N}}\left(I^{\mathcal{N}}\right)=\omega$.

We are left with the case of cuts $I$ such that $\operatorname{cf}^{\mathscr{\prime l}}(I)=\omega$. We require another definition here.

Definition 4.8 (Kirby and Paris [KP]). Let $I$ be a cut of $\mathscr{M}$ which is closed under addition and multiplication. $I$ is strong in $\mathscr{M}$ if for every parametrically definable function $f: \mathscr{M} \rightarrow \mathscr{M}$ there is an $n \in M \backslash I$ such that for all $a \in I$, either $f(a) \in I$ or else $f(a)>n$.

Lemma 4.9. The sentence "I is strong in $\mathscr{M}$ " is expressible in $(\mathscr{M}, I)$.

Proof. $I$ is strong in $\mathscr{M}$ if and only if

$$
(\mathscr{M}, I) \vDash \forall x \exists y\left[\neg I(y) \wedge \forall z\left(I(z) \rightarrow\left\{I\left((x)_{z}\right) \vee(x)_{z}>y\right\}\right)\right] .
$$

Lemma 4.10. Let $I$ be a cut of $\mathscr{M}$ such that $\operatorname{cf}^{\prime \prime}(I)=\omega$. Then $I$ is not cofinally extendible.

Proof. By Lemma 4.4, $\omega \in \operatorname{Def}(\mathscr{M}, I)$. Thus it suffices to show that $\omega$ is not cofinally extendible.

Case I. $\omega$ if strong in $\mathscr{M}$. Modify the proof of Lemma 4.7 as follows. Take $I=\omega$ and let $\left\langle a_{n}: n \in \omega\right\rangle$ be a sequence of elements of $M \backslash \omega$ which is coinitial in $M \backslash \omega$. Construct $\mathscr{M}(b), \mathscr{N}$ as before. Since $\mathscr{M}(b)$ does not fill $\omega$, then $\omega$ is the only candidate for a set $J \subseteq N$ such that $(\mathscr{M}, \omega)<(\mathscr{N}, J)$. But the function $f(j)=(b)_{j}$ for all $j \in|\mathscr{M}(b)|$ demonstrates that $\omega$ is not strong in $\mathscr{M}(b)$. So $\omega$ is not strong in $\mathscr{N}$ either, whence $(\mathscr{M}, \omega) \nprec(\mathscr{N}, \omega)$.

Case II. $\omega$ is not strong in $\mathscr{M}$. This time we have been unable to find a construction which does not fill $\omega$, so we will use a different argument. Our 
main problem is that if $\mathscr{M} \prec_{c} \mathscr{N}$ and $\mathscr{N}$ is countable and fills $\omega$, then there are $2^{\aleph_{0}}$ candidates for extensions of $\omega$.

Let the countable set $\mathscr{F}$ enumerate all functions $f: \omega \rightarrow M$ which are coded in $\mathscr{M}$, i.e. such that there exists a $b \in M$ satisfying $f(n)=(b)_{n}$ in $\mathscr{M}$ for all $n \in \omega$. Let $\mathscr{U}$ be a nonprincipal ultrafilter on $\omega$ and let $\mathscr{N}=\mathscr{M}^{\mathscr{F}} \mid \mathscr{U}$ be the corresponding Skolem-type ultrapower of $\mathscr{M}$ (where $N=\{\hat{f}: f \in \mathscr{F}\}$; here $\hat{f}$ denotes the equivalence class of $f$ as usual). For each $m \in M$ let $f^{m}$ denote the constant function such that $f^{m}(n)=m$ for all $n \in \omega$. Let $\delta: M \rightarrow N$ be defined by $\delta(m)=\widehat{f^{m}}$ for all $m \in M$. Then the Los theorem holds and $\delta$ is an elementary embedding of $\mathscr{M}$ into $\mathscr{N}$. We identify $\mathscr{M}$ with its image and say that $\mathscr{M} \prec \mathscr{N}$.

In fact we have $\mathscr{M} \prec_{c} \mathscr{N}$. For if $\hat{f} \in N$, take $b \in M$ such that $f(n)=(b)_{n}$ in $\mathscr{M}$ for all $n \in \omega$. Then $\mathscr{M} \vDash f(n)<b$ for all $n \in \omega$, whence $\mathscr{N} \vDash \hat{f}<b$.

$\mathscr{N}$ is a proper extension of $\mathscr{M}$; moreover, $\mathscr{N}$ fills $\omega$. Let $j \in \mathscr{F}$ be the identity mapping $j(n)=n$ for all $n \in \omega$. Then $\mathscr{N} \vDash \hat{j}>n$ for all $n \in \omega$, and $\mathscr{N} \vDash \hat{j}<m$ for all $m \in M \backslash \omega$.

Let $W \subseteq N$ be defined by

$$
W=\{\hat{f} \in N: \hat{f}<m \text { for all } m \in M \backslash \omega\} .
$$

Then $W$ is a cut of $N$ and $\omega \subsetneq W$; in fact $\hat{j} \in W$. Suppose $\Omega \subseteq N$ is a candidate for a subset of $N$ such that $(\mathscr{M}, \omega) \prec(\mathscr{N}, \Omega)$. Then $\Omega$ is a cut of $N$ and $\omega \subseteq \Omega \subseteq W$.

Our strategy now is to choose the ultrafilter $\mathscr{U}$ so as to eliminate all possibilities for $\Omega$ except for $\Omega=\omega$ and $\Omega=W$. We will then show that $(\mathcal{N}, \omega)$ and $(\mathscr{N}, W)$ are not elementary extensions of $(\mathscr{M}, \omega)$ and this will complete the proof.

Enumerate $\mathscr{F}$ as $\left\langle f_{n}: n \in \omega\right\rangle$. We will define a descending sequence $X_{n}$ $(n \in \omega)$ of infinite subsets of $\omega$ such that each $X_{n} \in \operatorname{SSy}(\mathscr{M})$-in other words, each $X_{n}$ is the intersection of $\omega$ with a parametrically definable subset of $M$.

Let $n \in \omega$ and suppose $X_{n-1}$ has been defined (take $X_{-1}=\omega$ ).

Case 1 . There is a $k \in \omega$ such that $f_{n}(i)=k$ for infinitely many $i \in X_{n-1}$.

Let $X_{n}=\left\{i \in X_{n-1}: f_{n}(i)=k\right\}$.

Case 2. There are only finitely many $i \in X_{n-1}$ such that $f_{n}(i) \in \omega$.

Let $Y_{n}=\left\{i \in X_{n-1}: f_{n}(i) \in M \backslash \omega\right\}$. Since $Y_{n}$ differs from $X_{n-1}$ only by a finite set, then $Y_{n}$ is infinite and $Y_{n} \in \operatorname{SSy}(\mathscr{M})$. Thus there is a nonstandard $a \in M \backslash \omega$ and an increasing sequence $\left\langle y_{i}: i \leq a\right\rangle$ coded in $\mathscr{M}$ such that $Y_{n}=\left\{y_{i}: i \in \omega\right\}$. Now for all $j \in \omega$,

$$
\mathscr{M} \vDash \forall i \leq j\left(f_{n}\left(y_{i}\right)>j\right) ;
$$

hence by Overspill there is a nonstandard $b \in M$ such that

$$
\mathscr{M} \vDash \forall i \leq b\left(f_{n}\left(y_{i}\right)>b\right) \text {. }
$$


Therefore we have shown that

$$
Y_{n}=\left\{i \in X_{n-1}: f_{n}(i)>b\right\} .
$$

Take $X_{n}=Y_{n}$ in this case.

Case 3. (The only remaining possibility.) Range $\left(f_{n} \uparrow X_{n-1}\right) \cap \omega$ is unbounded in $\omega$ but for each $k \in \omega, f_{n}^{-1}(\{k\}) \cap X_{n-1}$ is finite.

Let $S_{n-1} \in \operatorname{Def}(\mathscr{M})$ be such that $S_{n-1} \cap \omega=X_{n-1}$, and let $F_{n}: M \rightarrow M$ be a parametrically definable function on $M$ such that $F_{n} \mid \omega=f_{n}$. Define $G_{n}: M \rightarrow M$ to be the function $G_{n}(x)=F_{n}(x)+x$ for all $x \in M$, and let $g_{n}=G_{n} \mid \omega$. Then for all $m \in M, G_{n}(m) \geq m$ and $G_{n}(m) \geq F_{n}(m)$. Since $G_{n}$ is parametrically definable, $g_{n} \in \mathscr{F}$.

Now define $V_{n} \in \operatorname{Def}(\mathscr{M})$ as follows:

$$
V_{n}=\left\{m \in S_{n-1}: \mathscr{M} \vDash \forall x>m\left[S_{n-1}(x) \rightarrow\left(G_{n}(x)>G_{n}(m)\right)\right]\right\} .
$$

Let $Z_{n}=V_{n} \cap \omega$. Then $Z_{n} \subseteq X_{n-1}$ and $Z_{n} \in \operatorname{SSy}(\mathscr{M})$.

Claim 4.11. $Z_{n}$ is infinite.

Proof. Let $i \in X_{n-1}$ be such that $f_{n}(i) \in \omega$. Then $g_{n}(i) \in \omega$ as well; also $g_{n}(i) \geq i$. We will show that there is an element of $Z_{n}$ in the interval $\left[i, g_{n}(i)\right]$. Since there are by assumption infinitely many $i \in X_{n-1}$ such that $f_{n}(i) \in \omega$, this suffices.

Given $i \in X_{n-1}$ as above, let $c_{i}$ be the minimum value of the function $g_{n}$ on the finite set $X_{n-1} \cap\left[i, g_{n}(i)\right]$. Since $i \in X_{n-1}$, clearly $c_{i} \leq g_{n}(i)$. Let $d_{i} \in X_{n-1} \cap\left[i, g_{n}(i)\right]$ be maximal such that $g_{n}\left(d_{i}\right)=c_{i}$. We will show that $d_{i} \in Z_{n}$.

Now $d_{i} \in X_{n-1} \subseteq S_{n-1}$. Suppose $s \in S_{n-1}$ and $s>d_{i}$. If $s \leq g_{n}(i)$ then $s \in X_{n-1} \cap\left[i, g_{n}(i)\right]$, hence $g_{n}(s)>c_{i}=g_{n}\left(d_{i}\right)$ by definition of $c_{i}$ and $d_{i}$. If $s>g_{n}(i)$, then $G_{n}(s) \geq s>g_{n}(i) \geq c_{i}=g_{n}\left(d_{i}\right)$. Thus $d_{i} \in \omega$ is in $V_{n}$, so $d_{i} \in Z_{n}$.

Now $f_{n}(i) \in \omega$ for all $i \in Z_{n}$; this follows from the definitions and the hypotheses of Case 3. Let $\left\langle z_{i}: i \in \omega\right\rangle$ be an increasing enumeration of $Z_{n}$ which is coded in $\mathscr{M}$. Recursively define

$$
\begin{aligned}
& x_{0}=z_{0}, \\
& x_{k+1}=\mu z_{j}\left[z_{j}>x_{k} \wedge \forall i<j\left(f_{n}\left(z_{i}\right)<f_{n}\left(z_{j}\right)\right)\right] .
\end{aligned}
$$

The hypotheses of Case 3 guarantee that each $x_{k} \in \omega$. Let $X_{n}=\left\{x_{k}: k \in\right.$ $\omega\}$. Then $X_{n} \subseteq Z_{n}$ is infinite and $X_{n} \in \operatorname{SSy}(\mathscr{M})$. Also $f_{n}$ is 1-1 (in fact monotonically increasing) on $X_{n}$.

We have defined our descending sequence $X_{n} \quad(n \in \omega)$. The collection $\left\{X_{n}: n \in \omega\right\}$ has the finite intersection property so it generates a filter, which we extend arbitrarily to an ultrafilter $\mathscr{U}$.

Now $\bigcap\left\{X_{n}: n \in \omega\right\}=\varnothing$. For if $j \in \omega$ and $m$ is some arbitrary element of $M \backslash \omega$, then the function $f: \omega \rightarrow M$ defined by $f(j)=m$ and $f(i)=0$ for all 
$i \neq j$ was considered at some point, i.e., for some $n \in \omega$ we have $f=f_{n} \in \mathscr{F}$. Clearly $j \notin X_{n}$. Thus the ultrafilter $U$ must be nonprincipal.

Claim 4.12. Suppose $\Omega \subseteq N$ is such that $(\mathscr{M}, \omega) \prec(\mathscr{N}, \Omega)$ and suppose $\hat{j} \in \Omega$, where $j$ is the identity function on $\omega$. Then $\Omega=W$.

Proof. We have already noted that such an $\Omega$ must be a cut of $N$ satisfying $\omega \subseteq \Omega \subseteq W$. Suppose $\hat{f}_{n} \in W \backslash \omega$. Then $\hat{f}_{n}$ fills $\omega$ in the extension, so at stage $n$ the set $X_{n}$ must have been defined according to Case 3. By construction, then, Range $\left(f_{n} \mid X_{n}\right) \subseteq \omega$. But $X_{n} \in \operatorname{SSy}(\mathscr{M})$, hence there is some $f_{n^{\prime}} \in \mathscr{F}$ such that $f_{n^{\prime}}: \omega \rightarrow \omega$ and $f_{n^{\prime}} \mid X_{n}=f_{n} \uparrow X_{n} . X_{n} \in U$, hence $\hat{f}_{n^{\prime}}=\hat{f}_{n}$. Furthermore $\hat{f}_{n}=\hat{f}_{n^{\prime}}=f_{n^{\prime}}(\hat{j})$. Let $F_{n^{\prime}}: M \rightarrow M$ be parametrically definable in $\mathscr{M}$ such that $F_{n^{\prime}} \mid \omega=f_{n^{\prime}}$.

Now $(\mathscr{M}, \omega) \vDash \forall x\left[\omega(x) \rightarrow \omega\left(F_{n^{\prime}}(x)\right)\right]$. Therefore we must also have

$$
(\mathcal{N}, \Omega) \vDash \forall x\left[\Omega(x) \rightarrow \Omega\left(F_{n^{\prime}}(x)\right)\right] .
$$

By assumption $(\mathscr{N}, \Omega) \vDash \Omega(\hat{j})$, so $(\mathscr{N}, \Omega) \vDash \Omega\left(F_{n^{\prime}}(\hat{j})\right)$. That is to say, $(\mathscr{N}, \Omega) \vDash \Omega\left(\hat{f}_{n}\right)$. Since $\hat{f}_{n} \in W \backslash \omega$ was arbitrary and $\omega \subseteq \Omega \subseteq W$, then $\Omega=W$.

Claim 4.13. Suppose $\Omega \subseteq N$ is such that $(\mathscr{M}, \omega) \prec(\mathscr{N}, \Omega)$ and suppose $\Omega \neq \omega$. Then $\hat{j} \in \Omega$.

Proof. By assumption there is some $n \in \omega$ such that $\widehat{f}_{n} \in W \backslash \omega$ belongs to $\Omega$. As in the previous proof, it must be the case that $f_{n}$ satisfies the hypotheses of Case 3. By construction, then, Range $\left(f_{n} \mid X_{n}\right) \subseteq \omega$ and $f_{n}$ is 1-1 on $X_{n}$. Therefore if $U_{n}=\operatorname{Range}\left(f_{n} \mid X_{n}\right)$ then $U_{n} \subseteq \omega$ is infinite and $U_{n} \in \operatorname{SSy}(\mathscr{M})$. There is thus an $h_{n} \in \mathscr{F}$ such that for all $x \in X_{n}, h_{n}\left(f_{n}(x)\right)=x$. Since the only values of $h_{n}$ which matter are those it takes on $U_{n}$, we can find an $h_{n^{\prime}}: \omega \rightarrow \omega$ such that $h_{n^{\prime}} \in \mathscr{F}$ and $h_{n^{\prime}}\left|U_{n}=h_{n}\right| U_{n}$. Let $H_{n^{\prime}}: M \rightarrow M$ be parametrically definable in $\mathscr{M}$ such that $H_{n^{\prime}} \mid \omega=h_{n^{\prime}}$.

So $H_{n^{\prime}}\left(f_{n}(x)\right)=x$ for all $x \in X_{n}$. Thus $\mathscr{N} \vDash H_{n^{\prime}}\left(\hat{f}_{n}\right)=\hat{j}$. We have seen that

$$
(\mathscr{M}, \omega) \vDash \forall x\left[\omega(x) \rightarrow \omega\left(H_{n^{\prime}}(x)\right)\right] .
$$

Thus $(\mathcal{N}, \Omega) \vDash \forall x\left[\Omega(x) \rightarrow \Omega\left(H_{n^{\prime}}(x)\right)\right]$. By assumption $(\mathcal{N}, \Omega) \vDash \Omega\left(\hat{f}_{n}\right)$, so the above implies that $(\mathscr{N}, \Omega) \vDash \Omega\left(H_{n^{\prime}}\left(\hat{f}_{n}\right)\right)$, i.e. $(\mathscr{N}, \Omega) \vDash \Omega(\hat{j})$.

Claims 4.12 and 4.13 together imply that if $(\mathscr{M}, \omega) \prec(\mathcal{N}, \Omega)$, then either $\Omega=\omega$ or $\Omega=W$. The following claim completes the proof of Lemma 4.10.

Claim 4.14. (i) $(\mathscr{M}, \omega) \nprec(\mathscr{N}, \omega)$.

(ii) $(\mathscr{M}, \omega) \nprec(\mathscr{N}, W)$.

Proof. (i) Recall that we are assuming that $\omega$ is not strong in $\mathscr{M}$. Then there is a parametrically definable function $f: M \rightarrow M$ such that

$$
(\mathscr{M}, \omega) \vDash \neg \exists x[\neg \omega(x) \wedge \forall y(\omega(y) \rightarrow(\omega(f(y)) \vee f(y)>x))] .
$$


However, $(\mathscr{N}, \omega)$ satisfies the negation of the above sentence-take $x$ to be any element of $W \backslash \omega$.

(ii) Take $f$ as in (i). Then Range $(f \nmid \omega) \cap(M \backslash \omega)$ is coinitial in $M \backslash \omega$ in the model $\mathscr{M}$, therefore it is also coinitial in $N \backslash W$ in $\mathscr{N}$. Now if $k \in \omega$, then the range of $f\lceil[0, k]$ is clearly not coinitial in $M \backslash \omega$; that is,

$$
(\mathscr{M}, \omega) \vDash \neg \exists x[\omega(x) \wedge \forall y(\neg \omega(y) \rightarrow \exists z<x(\neg \omega f(z) \wedge f(z)<y))] .
$$

However, $(\mathscr{N}, W)$ is a model of the negation of the above sentence (with $W$ replacing $\omega$ ). Again we can take $x$ to be any element of $W \backslash \omega$, so in particular Range $(f \backslash[0, x]) \supseteq \operatorname{Range}(f\lceil\omega)$, hence its intersection with $N \backslash W$ is coinitial in $N \backslash W$.

We have proved Lemma 4.10 .

As we pointed out in the discussion following Theorem 4.2, we can combine Theorem 4.2 with Lemmas 4.7 and 4.10 to obtain

Theorem 4.15. Let $\mathscr{M} \vDash \mathrm{PA}$ be countable and nonstandard and let $U \subseteq M$. The following are equivalent:

(i) $U$ is cofinally extendible.

(ii) $U$ is uniquely cofinally extendible.

(iii) $U$ is inductive.

Furthermore, the implication (iii) $\Rightarrow$ (ii) (as well as (ii) $\Rightarrow$ (i), obviously) holds for arbitrary nonstandard $\mathscr{M}$.

We now provide a counterpart to Proposition 3.16.

Proposition 4.16. Let $\mathscr{M} \vDash \mathrm{PA}$ be countable and nonstandard. Then there is a subset $U \subseteq M$ which is not weakly cofinally extendible.

Proof. Let $c_{0}<c_{1}<\cdots<c_{n}<\cdots \quad(n \in \omega)$ be an increasing $\omega$-sequence cofinal in $\mathscr{M}$ and let $C=\left\{c_{n}: n \in \omega\right\}$. Let $g: C \rightarrow M$ be onto $M$, and take $U=\left\{\beta\left(c_{n}, g\left(c_{n}\right)\right): n \in \omega\right\}$.

Now $c_{n} \leq \beta\left(c_{n}, g\left(c_{n}\right)\right)$ for all $n \in \omega$, hence the elements of $U$ written in increasing order also form an $\omega$-sequence cofinal in $\mathscr{M}$. For every $a \in M$, $U \cap[0, a)$ is finite, hence parametrically definable in $\mathscr{M}$ so $U$ is a (proper) class.

Suppose $\mathscr{N}$ is a proper cofinal extension of $\mathscr{M}$, and suppose $V \subseteq N$ is such that $(\mathscr{M}, U) \prec(\mathscr{N}, V)$. Since $U$ is a class, there is only one possibility for $V$; in fact, for each $a \in M$ we must have $U \cap[0, a)=V \cap[0, a)$ since the former set is finite. But $\mathscr{M} \prec_{c} \mathscr{N}$, so it must be the case that $V=U$. Now

$$
(\mathscr{M}, U) \vDash \forall x \exists y\left(U(y) \wedge \pi_{2}(y)=x\right),
$$

whereas the elements of $N \backslash M$ are not in the range of $g$. Thus $(\mathscr{M}, U) \nprec$ $(\mathcal{N}, U)$.

The preceding proposition does not generalize to uncountable models. 
Proposition 4.17. Let $\mathscr{M} \vDash$ PA have uncountable cofinality. Then every $U \subseteq M$ is weakly cofinally extendible.

Proof. Let $D$ be a nonprincipal ultrafilter on $\omega$ and form the ultraproduct $\mathscr{M}^{*}=\mathscr{M}^{\omega} / D$. Then $\mathscr{M}^{*}$ is a proper elementary extension of $\mathscr{M}$, and $\mathscr{M}_{c}$ $\mathscr{M}^{*}$ because any countable sequence of elements of $M$ is bounded in $\mathscr{M}$. Let $V \subseteq\left|\mathscr{M}^{*}\right|$ be the set $U^{\omega} / D$; then $(\mathscr{M}, U) \prec\left(\mathscr{M}^{*}, V\right)$.

\section{Simple AND RECURSIVELY SATURATED EXTENDIBILITY}

In keeping with the pattern of the previous two sections, we begin with a modification of Definition 0.1 obtained by restricting the collection of elementary extensions to be considered.

Definition 5.1. Let $\mathscr{M} \vDash \mathrm{PA}, U \subseteq M . U$ is simply extendible if $U$ is extendible to $\mathscr{N}$ whenever $\mathscr{N}$ is a simple elementary extension of $\mathscr{M}$.

Then Theorem 0.3 can be strengthened:

Theorem 5.2. Let $\mathscr{M} \vDash \mathrm{PA}$ be countable, $U \subseteq M$. Then $U$ is simply extendible $\Leftrightarrow U \in \operatorname{Def}(\mathscr{M})$.

Proof. $(\Leftarrow)$ is clear from Proposition 0.2

$\Rightarrow$ By considering a simple proper conservative elementary end extension $\mathcal{N}$ of $\mathscr{M}$, we can strengthen Theorem 3.3 to show that if $U$ is a proper class in $\mathscr{M}$, then $U$ is not simply end extendible. (Here there are no restrictions on the cardinality of $\mathscr{M}$.)

We turn now to cofinal extendibility. Given a cut $I$ of $\mathscr{M}$ such that $\mathrm{cf}^{\mathscr{}}(I)$ $\neq \omega$, consider the model $\mathcal{N}$ constructed in the proof of Lemma 4.7. We see by the construction that there is an $e \in N$ such that for all $n \in \omega$ there is an $i \in \omega$ such that $\mathscr{N} \vDash(e)_{i}=a_{n}$. Then the simple extension $\mathscr{M}(e)$ of $\mathscr{M}$ has the following properties: $\mathscr{M} \prec_{c} \mathscr{M}(e), \mathscr{M}(e)$ does not fill $I$, and $\operatorname{cf}^{\mathscr{M}(e)}\left(I^{\mathscr{M}(e)}\right)=$ $\omega$. Thus $I$ is not simply cofinally extendible. A similar argument applies to Case I of Lemma 4.10.

In Case II of that lemma, the model $\mathscr{N}=\mathscr{M}^{\mathscr{T}} / \mathscr{U}$ is already a simple elementary cofinal extension of $\mathscr{M}$, for $\mathscr{N}=\mathscr{M}(\hat{j})$. To see this, let $\hat{f} \in N$ be arbitrary where $f \in \mathscr{F}$, and let $F: M \rightarrow M$ be parametrically definable in $\mathscr{M}$ such that $F \mid \omega=f$. Then $\mathcal{N} \vDash \hat{f}=F(\hat{j})$. We have thus shown that if $I$ is a cut of $\mathscr{M}$ such that $\mathrm{cf}^{\mathscr{}}(I)=\omega$, then $I$ is not simply cofinally extendible. As before, this completes the proof of the theorem.

Corollary 5.3. We can append two additional equivalent conditions to those of Theorem 4.15, namely:

(iv) $U$ is simply cofinally extendible.

(v) $U$ is uniquely simply cofinally extendible. 
Next we consider a different type of alteration of the definition of extendibility. This time, instead of making restrictions on the extension, we place our restriction on the models themselves.

Definition 5.4. Let $\mathscr{M} \vDash$ PA be recursively saturated and let $U \subseteq M . U$ is recursively saturated extendible (or r.s. extendible) if $U$ is extendible to $\mathscr{N}$ whenever $\mathscr{N}$ is recursively saturated and $\mathscr{M} \prec \mathscr{N}$.

Our main result goes through in this case as well.

Theorem 5.5. Let $\mathscr{M} \vDash \mathrm{PA}$ be countable and recursively saturated and let $U \subseteq$ $M$. Then $U$ is r.s. extendible $\Leftrightarrow U \in \operatorname{Def}(\mathscr{M})$.

Proof. Again $(\Leftrightarrow)$ is clear. To prove $(\Rightarrow)$, we first consider end extensions. We cannot appeal to the MacDowell-Specker Theorem this time; a result of $\mathrm{H}$. Kotlarski's shows that for any models $\mathscr{M}, \mathscr{N}$ of PA, if $\mathscr{N}$ is a proper conservative elementary end extension of $\mathscr{M}$ then $\mathscr{N}$ is not recursively saturated (see [KS].) However, M. Kaufmann proves in [Ka] that if $\mathscr{M} \vDash \mathrm{PA}$ is countable and recursively saturated and $U$ is a proper class in $\mathscr{M}$, then there is a countable recursively saturated $\mathscr{N}$ such that $\mathscr{M} \prec_{e} \mathscr{N}$ and there does not exist a class $V \subseteq N$ such that $V \cap M=U$. From the proof of Theorem 3.3 it is clear that $U$ is not extendible to $\mathscr{N}$. (Note: Kaufmann's lemma is generalized by J. Schmerl in [Sch] to recursively saturated $\mathscr{M}$ of countable cofinality.)

For cofinal extensions, the work has again been done for us. This time we simply quote the Smorynski-Stavi Theorem [SS]: Any cofinal extension of a recursively saturated model is again recursively saturated. Thus $\S 4$ carries over unchanged, and the theorem follows.

\section{OPEN QUESTIONS}

Our main open problem is the obvious one:

Question 6.1. Does Theorem 0.3 generalize to uncountable $\mathscr{M}$ ?

Theorem 3.14 reduces this to the equivalent question about Theorem 4.15; in turn, it would suffice to generalize Lemmas 4.7 and 4.10 to uncountable $\mathscr{M}$. Most of the available machinery for constructing cofinal extensions relies on the models being countable.

As we remarked before, we failed in $\S 3$ to obtain a complete characterization of the end extendible subsets of $\mathscr{M}$ for $\mathscr{M}$ nonstandard, or even for $\mathscr{M}$ countable and nonstandard. An answer to the following question would be helpful.

Question 6.2. Let $\mathscr{M} \vDash \mathrm{PA}$ be nonstandard. Is there an unbounded $U \subseteq M$, $U \notin \operatorname{Def}(\mathscr{M})$, such that $U$ is end extendible?

There are many questions we could ask about extendible subsets of arbitrary structures. It would be nice to find a structure $\mathscr{A}$ such that $\operatorname{Def}(\mathscr{A}) \subsetneq \mathscr{E}(\mathscr{A}) \subsetneq$ $\mathscr{P}(\mathscr{A})$ and for which we have a complete description of $\mathscr{E}(\mathscr{A})$. This leads us to ask the following. 
Question 6.3. Let $\mathscr{A}=\langle\mathscr{A},<\rangle$ be a dense linear ordering without endpoints. Is there a nice characterization of $\mathscr{E}(\mathscr{A})$ ?

Our examples in $\S 2$ suggest an inverse correlation between the strength of a theory $T$ and the size of $\mathscr{E}(\mathscr{M})$ (relative to $\operatorname{Def}(\mathscr{M})$ and $\mathscr{P}(\mathscr{M})$ ). We would like to make this idea more precise somehow.

Question 6.4. Is there a theory $T$ such that $\mathscr{E}(\mathscr{A})=\operatorname{Def}(\mathscr{A})$ for all $\mathscr{A} \vDash T$ ? Is PA such a theory? If a theory $T$ has an infinite model $\mathscr{B}$ such that $\mathscr{E}(\mathscr{B})=$ $\operatorname{Def}(\mathscr{B})$, does $\mathscr{E}(\mathscr{A})=\operatorname{Def}(\mathscr{A})$ for all $\mathscr{A} \vDash T$ ?

Question 6.5. The same question (excluding the second part about PA) with $\mathscr{P}(\mathscr{A})$ replacing $\operatorname{Def}(\mathscr{A})$ and $\mathscr{P}(\mathscr{B})$ replacing $\operatorname{Def}(\mathscr{B})$.

Finally, we mention the following question which arose during the preparation of $\S 4$.

Question 6.6. Let $\mathscr{M} \vDash$ PA be nonstandard and let $I$ be a cut of $\mathscr{M}$ such that $I \in \operatorname{Def}(\mathscr{M}, \omega)$. Does $\mathrm{cf}^{\mathscr{\prime}}(I)=\omega$ ?

A negative answer for all countable $\mathscr{M}$ would eliminate the need for Lemma 4.10. A positive answer for all $\mathscr{M}$ would give us a nice recriprocal definability result, namely: Let $\mathscr{M} \vDash$ PA be nonstandard and suppose

$$
U \in \operatorname{Def}(\mathscr{M}, \omega) \backslash \operatorname{Def}(\mathscr{M}) .
$$

Then $\omega \in \operatorname{Def}(\mathscr{M}, U)$. [The proof would go as follows: By Corollary 3.15, $U$ is not a proper class in $\mathscr{M}$, hence $U$ is not inductive. Therefore, there is a cut $I$ of $\mathscr{M}$ such that $I \in \operatorname{Def}(\mathscr{M}, U)$. Then $I \in \operatorname{Def}(\mathscr{M}, \omega)$, so our positive answer to Question 6.6 tells us that $\mathrm{cf}^{\mathscr{\prime}}(I)=\omega$. By Lemma 4.4, $\omega \in \operatorname{Def}(\mathscr{M}, I)$, and since $I \in \operatorname{Def}(\mathscr{M}, U)$ we conclude that $\omega \in \operatorname{Def}(\mathscr{M}, U)$.

\section{REFERENCES}

[BS] J. Barwise and J. Schlipf, An introduction to recursively saturated and resplendent models, J. Symbolic Logic 41 (1976), 531-536.

[CK] C. C. Chang and H. J. Keisler, Model theory, North-Holland, Amsterdam, 1973.

[G] H. Gaifman, A note on models and submodels of arithmetic (Conference in Mathematical Logic, London 1970; W. Hodges, ed.), Lecture Notes in Math., vol. 255. Springer-Verlag. Heidelberg, 1972, pp. 128-144.

[Ka] M. Kaufmann, A rather classless model, Proc. Amer. Math. Soc. 62 (1977), 330-333.

[KS] M. Kaufmann and J. Schmerl, Saturation and simple extensions of models of Peano Arithmetic, Ann. Pure Appl. Logic 27 (1984), 109-136.

[Ki] L. Kirby, Initial segments of models of arithmetic, Thesis, Manchester University, 1977.

[KP] L. Kirby and J. Paris, Initial segments of models of Peano's axioms (Set Theory and Hierarchy Theory V. Bierutowice 1976), Lecture Notes in Math., vol. 619, Springer-Verlag, Heidelberg. 1977, pp. $211-226$.

[Ko] H. Kotlarski, On cofinal extensions of models of arithmetic, J. Symbolic Logic 40 (1983), pp. 253-262. 
[MS] R. MacDowell and E. Specker, Modelle der Arithmetik, Infinitistic Methods (Proceedings of the Symposium on the Foundations of Mathematics, Warsaw 1959), Pergamon Press, London, 1961, pp. 257-263.

[Sch] J. Schmerl, Recursively saturated, rather classless models of Peano arithmetic (Logic Year 1979-80: The University of Connecticut; M. Lerman. J. Schmerl, and R. Shore, eds.), Lecture Notes in Math., vol. 859, Springer-Verlag, Heidelberg, 1981, pp. 268-282.

[Smol] C. Smorynski, Cofinal extensions of nonstandard models of arithmetic, Notre Dame J. Formal Logic 22 (1981), 133-144.

[Smo2] __, Recursively saturated nonstandard models of arithmetic, J. Symbolic Logic 46 (1981), 259-286.

[Smo3] __, Back-and-forth inside a recursively saturated model of arithmetic (Logic Colloquium '80; D. van Dalen, D. Lascar, and J. Smiley, eds.), Studies in Logic and the Foundations of Mathematics, Vol. 108, North-Holland, Amsterdam, 1982, pp. 273-278.

[SS] C. Smorynski and J. Stavi, Cofinal extension preserves recursive saturation (Model Theory of Algebra and Arithmetic: Proceedings, Karpacz, Poland, 1979; L. Pacholski, J. Wierzejewski, and A. J. Wilkie, eds.), Lecture Notes in Math., vol. 834, Springer-Verlag, Heidelberg, 1980, pp. 338-345.

Department of Mathematics and Computer Sciences, Ben Gurion University of the Negev, Beersheva, IsRael 